Pure and Applied Mathematics Quarterly

Volume 2, Number 2

(Special Issue: In honor of

John H. Coates, Part 2 of 2$)$

$569-599,2006$

\title{
Arithmetic Fuchsian Groups of Genus Zero
}

\author{
D. D. Long, C. Maclachlan and A. W. Reid
}

\section{INTRODUCTION}

If $\Gamma$ is a finite co-area Fuchsian group acting on $\mathbf{H}^{2}$, then the quotient $\mathbf{H}^{2} / \Gamma$ is a hyperbolic 2-orbifold, with underlying space an orientable surface (possibly with punctures) and a finite number of cone points. Through their close connections with number theory and the theory of automorphic forms, arithmetic Fuchsian groups form a widely studied and interesting subclass of finite co-area Fuchsian groups. This paper is concerned with the distribution of arithmetic Fuchsian groups $\Gamma$ for which the underlying surface of the orbifold $\mathbf{H}^{2} / \Gamma$ is of genus zero; for short we say $\Gamma$ is of genus zero.

The motivation for the study of these groups comes from many different viewpoints. For example, a classical situation, with important connections to elliptic curves is to determine which subgroups $\Gamma_{0}(n)$ of the modular group have genus zero. It is not difficult to show that the only possible values are $1 \leq n \leq 10,12$, 13, 16, 18 and 25. In addition, J. G. Thompson [43] showed that only finitely many congruence subgroups have genus 0 . On the otherhand, it is easy to see that there are subgroups of genus 0 inside the modular group of arbitrary large finite index. For if $\Gamma(2)$ denotes the principal congrence subgroup of level 2 in $\operatorname{PSL}(2, \mathbb{Z})$ (see $\S 4.1$ ), then $\mathbf{H}^{2} / \Gamma(2)$ is a sphere with 3 -punctures, and it is easy to construct an $\mathrm{m}$-fold cyclic cover of $\mathbf{H}^{2} / \Gamma(2)$ where two of the punctures have a single connected pre-image, and the last lifts to $\mathrm{m}$ punctures. The groups corresponding to these covers all have genus 0 .

In addition, it is well-known that there are cocompact arithmetic Fuchsian groups of genus 0 (eg certain triangle groups [41]) and indeed it is not difficult to construct infinitely many cocompact arithmetic Fuchsian groups of genus 0 (see Corollary 4.10). The main result of this paper is:

Received January 7, 2006.

The first author's work was partially supported by the N. S. F. The third author's work was partially supported by the N. S. F. and a grant from the Texas Advanced Research Program. 
Theorem 1.1. There are finitely many conjugacy classes of congruence arithmetic Fuchsian groups of genus zero. There are thus finitely many conjugacy classes of maximal arithmetic Fuchsian groups of genus 0 . There are then finitely many (wide) commensurability classes which contain arithmetic Fuchsian groups of genus zero.

Particular cases of genus zero Fuchsian groups arise as the index 2 subgroup consisting of orientation-preserving isometries in the group generated by reflections in the faces of a polygon in $\mathbf{H}^{2}$ all of whose interior angles are integer submultiples of $\pi$. Hence, as a corollary of Theorem 1.1 we can prove the following strengthening of the results of Nikulin [33] in the case of dimension 2. (See [2] for similar results in dimension 3.)

Corollary 1.2. There are finitely many (wide) commensurability classes of arithmetic reflection groups acting on $\mathbf{H}^{2}$.

Cocompact Fuchsian groups of genus 0 have abelianizations that are finite, and so are the 2-dimensional analogues of rational homology 3 -spheres. If we restrict attention to arithmetic Fuchsian groups which have trivial abelianization (so analogues of integral homology 3 -spheres) we obtain:

Theorem 1.3. The only arithmetic Fuchsian groups with trivial abelianizations are the $(2,3,7)$ and $(2,3,11)$ triangle groups.

In this paper we also construct genus 0 arithmetic Fuchsian groups arising from defining fields of degrees $\leq 7$, and make a detailed study of the case when the defining field is $\mathbb{Q}$. In this case we give a complete list of maximal arithmetic Fuchsian groups of genus 0, including the non-cocompact setting (some of this has already appeared, see [36, 37, 10] and [20] for example).

To conclude the paper we discuss some comparisons with the 3-dimensional setting. For example, recall that if $M=\mathbf{H}^{3} / \Gamma$ is a closed orientable hyperbolic 3-manifold, the injectivity radius of $M$ (denoted by injrad $(M)$ ) is defined as the largest number $r$ such that for all $p \in M$, the ball $B(p, r)$ centered at $p$ of radius $r$ is isometric to the $r$-ball in $\mathbf{H}^{3}$. The following question of Cooper has attracted some interest recently.

Question 1.4. Let $M$ be a closed hyperbolic 3-manifold which is a rational homology 3-sphere (i.e. the first betti number of $M$ is 0). Does there exist a $K \in \mathbb{R}$, independent of $M$, such that $\operatorname{inj} \operatorname{rad}(M) \leq K$ ?

Using residual finiteness, an affirmative answer to this question implies every closed hyperbolic 3-manifold has a cover with first betti number at least 1 (see [22] for more on this). Very recently, F. Calegari and N. Dunfield [7] have answered this question in the negative. They construct arithmetic rational homology 3-spheres arising from the division algebra over $\mathbb{Q}(\sqrt{-2})$ ramified at the 
two places lying above 3 in $\mathbb{Q}(\sqrt{-2})$ and with arbitrarily large injectivity radii. The construction of [7] assumes the existence of certain galois representations of $\operatorname{Gal}(\overline{\mathbb{Q}(\sqrt{-2})} / \mathbb{Q}(\sqrt{-2}))$ that although predicted by the general framework of the Langlands correspondence are not yet known to exist. However an unconditional proof of this has now been established in [6].

With this in mind, we prove the following result in dimension 2.

Theorem 1.5. Let $F=\mathbf{H}^{2} / \Gamma$ be a closed orientable hyperbolic 2-orbifold, with all torsion elements of $\Gamma$ order $\leq p$. Suppose that the underlying topological surface of $F$ is a sphere or a torus.

Then there is a number $K=K(p)$ so that $F$ contains a geodesic of length at most $K$.

In particular, $K$ does not depend upon how many orbifold points there are, only on the maximal order of an elliptic element. Thus, Theorems 1.1 and 1.5 easily show that the 2-dimensional analogue of the result of Calegari and Dunfield does not hold (see Corollary 4.13).

Acknowledgments: We are very grateful to Nathan Dunfield for interesting email correspondences (see in particular the Remark at the end of $\S 4$ ), and for pointing out an omission in Table 2d. The third author wishes to thank Peter Sarnak for many useful conversations and correspondences on topics related to the work here, and in particular for pointing out [47].

\section{NUMBER THEORETIC PRELIMINARIES}

We will make use of results and definitions from number theory (see [21] and [31] for details) and the theory of quaternion algebras (see [27] or [44] for details)

2.1. By a number field $k$ we mean a finite extension of $\mathbb{Q}$. The ring of integers of $k$ will be denoted $R_{k}$. A place $\nu$ of $k$ will be one of the canonical absolute values of $k$ defined in [21], p.34 - 35. The finite places of $k$ correspond bijectively to the prime ideals of $R_{k}$. An infinite place of $k$ is either real, corresponding to an embedding of $k$ into $\mathbb{R}$, or complex, corresponding to a pair of distinct complex conjugate embeddings of $k$ into $\mathbb{C}$. We refer the reader to [21], p.36 for the definition of the completion $k_{\nu}$ of $k$ at a place $\nu$. When $\nu$ is an infinite place, $k_{\nu}$ is isomorphic to $\mathbb{R}$ or $\mathbb{C}$ depending on whether $\nu$ is real or complex.

If $\mathcal{A}$ is an ideal of $R_{k}$, the norm of $\mathcal{A}$ is the cardinality of the quotient ring $R_{k} / \mathcal{A}$ and will be denoted $\mathrm{N} \mathcal{A}$. 
2.2. Let $k$ be a field of characteristic different from 2 . The standard notation for a quaternion algebra over $\mathrm{k}$ is the following. Let $a$ and $b$ be non-zero elements of $k$. Then $\left(\frac{a, b}{k}\right)$ denotes the quaternion algebra over $k$ with basis $\{1, i, j, i j\}$ subject to $i^{2}=a, j^{2}=b$ and $i j=-j i$.

Let $k$ be a number field, and $\nu$ a place of $k$. If $B$ is a quaternion algebra defined over $k$, the classification of quaternion algebras $B_{\nu}=B \otimes_{k} k_{\nu}$ over the local fields $k_{\nu}$ is quite simple. If $\nu$ is complex then $B_{\nu}$ is isomorphic to $M\left(2, k_{\nu}\right)$ over $k_{\nu}$. Otherwise there is, up to isomorphism over $k_{\nu}$, a unique quaternion division algebra over $k_{\nu}$, and $B_{\nu}$ is isomorphic over $k_{\nu}$ to either this division algebra or to $M\left(2, k_{\nu}\right)$.

Let $B$ be a quaternion algebra over the number field $k$. B is ramified at a place $\nu$ of $k$ if $B_{\nu}$ is a division algebra. Otherwise we say $B$ is unramified at $\nu$. We shall denote the set of places (resp. finite places) at which $B$ is ramified by $\operatorname{Ram}(B)$ $\left(\right.$ resp. $\left.\operatorname{Ram}_{f}(B)\right)$. The discriminant of $B$ is the $R_{k}$-ideal $\prod_{\nu \in \operatorname{Ram}_{f}(B)} \mathcal{P}_{\nu}$ where $\mathcal{P}_{\nu}$ is the prime ideal associated to the place $\nu$.

We summarize for convenience the classification theorem for quaternion algebras over number fields (see [27] Chapter 7 or [44] Chapter 3).

Theorem 2.1. - The set $\operatorname{Ram}(B)$ is finite, of even cardinality and contains no complex places.

- Conversely, suppose $S$ is a finite set of places of $k$ which has even cardinality and which contains no complex places. Then there is a quaternion algebra $B$ over $k$ with $\operatorname{Ram}(B)=S$, and this $B$ is unique up to isomorphism over $k$.

- $B$ is a division algebra of quaternions if and only if $\operatorname{Ram}(B) \neq \emptyset$.

If $B$ is a quaternion algebra over $k$, and $K$ is an extension field of $k$, then $K$ is called a splitting field for $B$ if $B \otimes_{k} K \cong M(2, K)$. There is the following classification of quadratic extensions of $k$ that are splitting fields for $B$ (see [27] Chapter 7 or [44] Chapter 3).

Theorem 2.2. Let $B$ be a quaternion algebra over the number field $k$ and $K a$ quadratic extension of $k$. The following are equivalent:

- $K$ is a splitting field for $B$;

- $K$ embeds in $B$;

- if $\nu \in \operatorname{Ram}(B)$, then $\nu$ is not split in $K$.

\section{Arithmetic Fuchsian and Kleinian groups}

We recall the definition and some properties of arithmetic Fuchsian and Kleinian groups (see [27] for details). 
3.1. Let $k$ be a totally real number field of degree $n$, and let $B$ be a quaternion algebra defined over $k$ which is ramified at all infinite places except one. Let $\rho: B \rightarrow M(2, \mathbb{R})$ be an embedding, $\mathcal{O}$ be an order of $B$, and $\mathcal{O}^{1}$ the elements of norm one in $\mathcal{O}$. Then $\operatorname{P} \rho\left(\mathcal{O}^{1}\right)<\operatorname{PSL}(2, \mathbb{R})$ is a finite co-area Fuchsian group, which is co-compact if and only if $B$ is not isomorphic to $M(2, \mathbb{Q})$. A Fuchsian group $\Gamma$ is defined to be arithmetic if and only if $\Gamma$ is commensurable with some such $\mathrm{P} \rho\left(\mathcal{O}^{1}\right)$. Furthermore, $\Gamma$ is called derived from a quaternion algebra if $\Gamma<$ $\mathrm{P} \rho\left(\mathcal{O}^{1}\right)$.

Notation: Let $B / k$ be as above and $\mathcal{O}$ be an order of $B$. We will denote the group $\mathrm{P} \rho\left(\mathcal{O}^{1}\right)$ by $\Gamma_{\mathcal{O}}^{1}$.

Arithmetic Kleinian groups are obtained in a similar way. In this case we let $k$ be a number field having exactly one complex place, and $B$ a quaternion algebra over $k$ which is ramified at all real places of $k$. As above, if $\mathcal{O}$ is an order of $B$ and $\rho: \mathcal{O}^{1} \hookrightarrow \operatorname{SL}(2, \mathbb{C})$, then $\mathrm{P} \rho\left(\mathcal{O}^{1}\right)$ is a Kleinian group of finite covolume. An arithmetic Kleinian group $\Gamma$ is a subgroup of $\operatorname{PSL}(2, \mathbb{C})$ commensurable with a group of the type $\mathrm{P} \rho\left(\mathcal{O}^{1}\right)$. As above, $\Gamma$ is derived from a quaternion algebra if $\Gamma$ is actually a subgroup of some $\mathrm{P} \rho\left(\mathcal{O}^{1}\right)$. An arithmetic Kleinian group is cocompact if and only if the quaternion algebra $B$ as above is a division algebra.

In both the Fuchsian and Kleinian cases, the isomorphism class of the quaternion algebra $B / k$ determines a wide commensurability class of groups in $\operatorname{PSL}(2, \mathbb{R})$ and PSL $(2, \mathbb{C}$ ) respectively (see [27] Chapter 8). From the classification Theorem 2.1 for quaternion algebras over number fields the isomorphism classes of such quaternion algebras will be completely determined by the finite set of places of $\mathrm{k}$ at which $B$ is ramified.

3.2. As was shown by Borel [5] there are infinitely many maximal groups in the commensurability class of an arithmetic Fuchsian or Kleinian group. The description of these maximal groups was reformulated in [8] (see also [27] Chapter 11) using Eichler orders as follows:

Let $k$ be a number field and $B$ a quaternion algbera over $k$. An Eichler order $\mathcal{E}$ is defined to be the intersection of two maximal orders $\mathcal{O}, \mathcal{O}^{\prime}$. Let $S$ be the finite set of finite places $\nu$ of $k$ such that $\mathcal{O}_{\nu}:=\mathcal{O} \otimes_{R_{k}} R_{k_{\nu}}$ is not equal to $\mathcal{O}_{\nu}^{\prime}$. Necessarily $S \cap \operatorname{Ram}_{f}(B)=\emptyset$ and for $\nu \in S$, there exists a positive integer $n_{\nu}$ such that $\mathcal{E}_{\nu}=\mathcal{O}_{\nu} \cap \mathcal{O}_{\nu}^{\prime}$ is conjugate to

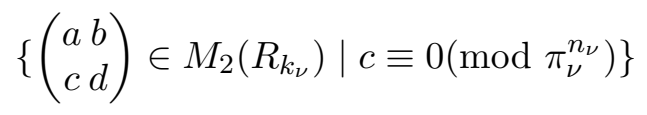

where $\pi_{\nu}$ is a uniformizer of $k_{\nu}$. The level of $\mathcal{E}$ is the set (or the product) of ideals $\mathcal{P}_{\nu}^{n_{\nu}}, \nu \in S$. If, for each $\nu \in S, n_{\nu}=1$ then $\mathcal{E}$ is said to be of square-free level $S$. 
Now suppose that $k$ is totally real, $B$ is ramified at all real places except one with $\rho: B \rightarrow M_{2}(\mathbb{R})$ and $\mathcal{E}$ is an Eichler order in $B$. Let

$$
N(\mathcal{E})=\left\{x \in B^{*} \mid x \mathcal{E} x^{-1}=\mathcal{E}\right\}
$$

and denote $P(\rho(N(\mathcal{E}))) \subset \operatorname{PGL}(2, \mathbb{R})$ by $\Gamma_{\mathcal{E}}$. Let $\Gamma_{\mathcal{E}}^{+}=\Gamma_{\mathcal{E}} \cap \operatorname{PSL}(2, \mathbb{R})$. Note that $\Gamma_{\mathcal{E}}^{+}$contains $\Gamma_{\mathcal{E}}^{1}$ as a subgroup of finite index.

Theorem 3.1 (Borel). Let $\Gamma$ be an arithmetic Fuchsian group in the commensurability class determined by the quaternion algebra $B$. Then $\Gamma$ is conjugate to a subgroup of some $\Gamma_{\mathcal{E}}^{+}$for $\mathcal{E}$ an Eichler order of square-free level.

Note that here we include the cases where $\mathcal{E}$ is a maximal order i.e. the set $S$ is empty.

Example 3.2. In the non-cocompact case, $B=M_{2}(\mathbb{Q})$ and all arithmetic Fuchsian groups are commensurable with $\operatorname{PSL}(2, \mathbb{Z})$. Each Eichler order is conjugate to

$$
\mathcal{E}_{0}(n):=\left\{\left(\begin{array}{ll}
a & b \\
c & d
\end{array}\right) \in M_{2}(\mathbb{Z}) \mid c \equiv 0(\bmod n)\right\}
$$

and the conjugacy classes of the maximal Fuchsian groups are the groups $\Gamma_{\mathcal{E}_{0}(n)}^{+}$ for $n$ square-free, which are familiar as the normalizers in $\operatorname{PSL}(2, \mathbb{R})$ of the groups $\Gamma_{0}(n)$.

\section{Proofs}

4.1. Recall that a congruence subgroup of $\operatorname{PSL}(2, \mathbb{Z})$ is any subgroup of $\operatorname{PSL}(2, \mathbb{Z})$ that contains a principal congruence subgroup $\Gamma(n)$ of level $n$ for some $n$ where

$$
\Gamma(n)=\mathrm{P}\{A \in \mathrm{SL}(2, \mathbb{Z}): A \equiv I \bmod n\} .
$$

More generally, a non-cocompact arithmetic Fuchsian group $\Gamma$ is congruence if some conjugate of $\Gamma$ in $\operatorname{PGL}(2, \mathbb{R})$ contains some $\Gamma(n)$. From the discussion of the non-cocompact case in Example 3.2, it follows that all maximal arithmetic Fuchsian groups commensurable with the modular group are congruence.

We need to extend this definition to the cocompact setting. To this end, let $B$ be a quaternion algebra over a totally real field $k$ and let $\mathcal{O}$ be a maximal order of $B$. Let $I$ be an integral 2 -sided $\mathcal{O}$-ideal in $B$ which means that $I$ is a complete $R_{k}$-lattice in $B$ such that

$$
\mathcal{O}=\{x \in B \mid x I \subset I\}=\{x \in B \mid I x \subset I\} .
$$

The principal congruence subgroup of $\Gamma_{\mathcal{O}}^{1}$ of level $I$, denoted $\Gamma_{\mathcal{O}}^{1}(I)$ is the image in $\operatorname{PSL}(2, \mathbb{R})$ of

$$
\mathcal{O}^{1}(I)=\left\{\alpha \in \mathcal{O}^{1}: \alpha-1 \in I\right\} .
$$

Since $\mathcal{O} / I$ is a finite ring, $\mathcal{O}^{1}(I)$ has finite index in $\mathcal{O}^{1}$. 
Definition 4.1. Let $\Gamma$ be an arithmetic Fuchsian group. $\Gamma$ is called a congruence subgroup if there is a quaternion algebra $B$ defined over the totally real field $k$ ramified at all real places except one, a maximal order $\mathcal{O}$ and an ideal $I$ of $\mathcal{O}$ as described above such that $\Gamma$ contains $\Gamma_{\mathcal{O}}^{1}(I)$.

Lemma 4.2. Any maximal arithmetic Fuchsian group is a congruence subgroup.

Proof: From Theorem 3.1, it suffices to show that $\Gamma_{\mathcal{E}}^{1}$ is a congruence subgroup where $\mathcal{E}$ is an Eichler order of square-free level $S$. Let $\mathcal{E}=\mathcal{O} \cap \mathcal{O}^{\prime}$. Define $I$ by locally ascribing $I_{\nu}$ to be the 2-sided $\mathcal{O}_{\nu}$-ideal $\pi_{\nu} \mathcal{O}_{\nu}$ for $\nu \in S$ and $I_{\nu}=\mathcal{O}_{\nu}$ for $\nu \notin S$. From the description at (1), it follows that, if $\alpha \in \mathcal{O}^{1}$ and $\alpha-1 \in I_{\nu}$ then $\alpha \in \mathcal{E}_{\nu}^{1}$. It then follows by local-global arguments that $\mathcal{O}^{1}(I) \subset \mathcal{E}^{1}$ (see e.g. [27, Chap 6]).

4.2. Proof of Theorem 1.1. By Lemma 4.2, the second part of the Theorem will follow from the first. Also, if a commensurability class contains an arithmetic Fuchsian group $\Gamma$ of genus zero, then any Fuchsian group containing $\Gamma$ will also have genus zero, so the last part of the theorem follows from the second. Thus assume we have an infinite sequence $\left\{\Gamma_{i}\right\}$ of non-conjugate congruence arithmetic Fuchsian groups of genus 0 . Then the Area $\left(\mathbf{H}^{2} / \Gamma_{i}\right) \rightarrow \infty$ as there are only finitely many conjugacy classes of arithmetic Fuchsian groups of bounded area (see [5] [42]).

We now make use of the following important result about congruence subgroups (see [15], [18] and [45]).

Theorem 4.3. If $\Gamma$ is a congruence arithmetic Fuchsian group then

$$
\lambda_{1}(\Gamma) \geq \frac{3}{16}
$$

where $\lambda_{1}(\Gamma)$ is the first non-zero eigenvalue of the Laplacian of $\Gamma$.

On the other hand, we have the following remarkable result of P. Zograf [47].

Theorem 4.4. Let $\Gamma$ be a Fuchsian group of finite co-area and let the genus of $\mathbf{H}^{2} / \Gamma$ be denoted by $g(\Gamma)$. If Area $\left(\mathbf{H}^{2} / \Gamma\right) \geq 32 \pi(g(\Gamma)+1)$, then

$$
\lambda_{1}(\Gamma)<\frac{8 \pi(g(\Gamma)+1)}{\operatorname{Area}\left(\mathbf{H}^{2} / \Gamma\right)} .
$$

We now have a contradiction, for with $g\left(\Gamma_{i}\right)=0$, and Area $\left(\mathbf{H}^{2} / \Gamma_{i}\right) \rightarrow \infty$, Theorem 4.4 implies that $\lambda_{1} \rightarrow 0$. However this contradicts Theorem 4.3.

The inequalities in Theorems 4.3 and 4.4 give an upper bound on the co-area of a maximal (resp. congruence) arithmetic Fuchsian group of genus zero as:

$$
\operatorname{Area}\left(\mathbf{H}^{2} / \Gamma\right) \leq \frac{128}{3} \pi
$$


This inequality together with the data defining the maximal arithmetic group can be used to determine those of genus zero and for groups defined over $\mathbb{Q}$, a complete enumeration is carried out in $\S 5$.

Corollary 4.5. There is an upper bound to the order of an elliptic element in an arithmetic Fuchsian group of genus 0.

Proof: Since there are only finitely many commensurability classes of arithmetic Fuchsian groups of genus 0 , there is a bound on the degree of the defining fields $k$ of these groups. If an arithmetic Fuchsian group contains an element of order $n \geq 2$, then $k$ contains $\mathbb{Q}(\cos 2 \pi / n)$ and it is well-known that the degree of this field goes to infinity with $n$.

Remarks:(1) The same arguments hold for arbitrary fixed $g$.

(2) It was previously known that there were finitely many $n$ such that the groups $N\left(\Gamma_{0}(n)\right)$, where $N$ indicates the normalizer in $\operatorname{PSL}(2, \mathbb{R})$, had genus 0 (see [36] and [37]). Intriguingly, these values of $\mathrm{n}$ are related to the properties of the Monster simple group (see [10] and [20] for more). For example Ogg (cf. [35]) observed that that those primes $p$ for which the groups $N\left(\Gamma_{0}(p)\right)$ have genus 0 , coincides with those primes $p$ dividing the order of the Monster simple group (see Table 1).

4.3. Our results can be interpreted in terms of Shimura curves (see [40] and also [1] for Shimura curves over $\mathbb{Q}$ ). By a Shimura curve we mean a quotient $\mathbf{H}^{2} / \Gamma_{\mathcal{E}}^{1}$ where $\mathcal{E}$ is an Eichler order (not necessarily of square free level).

Corollary 4.6. There are only finitely many Shimura curves of genus zero.

Proof: A mild adaptation of the argument in Lemma 4.2 shows that $\Gamma_{\mathcal{E}}^{1}$ is a congruence group even when $\mathcal{E}$ is not of square-free level.

4.4. Proof of Theorem 1.3. For convenience we make the following definition (motivated by the 3-dimensional situation).

Definition 4.7. Let $\Gamma$ be a Fuchsian group of finite co-area. We will say that $\mathbf{H}^{2} / \Gamma$ is an integral homology 2-sphere if $\Gamma^{\mathrm{ab}}=1$.

Note that $\Gamma$ is necessarily cocompact. For if not, then $\Gamma$ is the free product of a finite number of cyclic groups and the abelianization is non-trivial. With this definition, Theorem 1.3 follows from:

Theorem 4.8. The only arithmetic integral homology 2-spheres are the quotients of $\mathbf{H}^{2}$ by the $(2,3,7)$ and $(2,3,11)$ triangle groups. 
That the aforementioned triangle groups are arithmetic groups can be found in [41].

The proof will require the following lemma (for notation, see $\S 5$ below).

Lemma 4.9. Let $Q=\mathbf{H}^{2} / \Gamma$ be an integral homology 2-sphere. Then the group $\Gamma$ has signature $\left(0 ; m_{1}, \ldots, m_{r} ; 0\right)$ and the $m_{i}$ are pairwise coprime.

Proof: Since $\Gamma$ is cocompact and of genus zero, it has a presentation as given at (3) with $g=t=0$. Suppose the $m_{i}$ are not pairwise coprime. Then, after reordering if necessary, let the greatest common divisor of $m_{1}, m_{2}$ be $m$. We construct a homomorphism from $\Gamma$ onto $\mathbf{Z} / m \mathbf{Z}$, and obtain a contradiction. Let $\mathbf{Z} / m \mathbf{Z}=\langle\alpha>$. Define a homomorphsim $\phi: \Gamma \rightarrow\langle\alpha\rangle$ by:

$$
\phi\left(x_{i}\right)=\left\{\begin{array}{rl}
\alpha & i=1 \\
\alpha^{-1} & i=2 \\
1 \quad i & =3,4, \ldots, r
\end{array}\right.
$$

The result now follows.

Note that the lemma has the following easy corollary that provides lots of genus 0 arithmetic Fuchsian groups (e.g. apply the corollary to the $(2,4,6)$ triangle group).

Corollary 4.10. If $\Gamma$ has signature of the form $\left(0 ; m_{1}, m_{2}, \ldots, m_{r} ; 0\right)$ where the $m_{i}$ are not pair-wise coprime, then there are infinitely many subgroups of $\Gamma$ with signatures of the same form.

Proof: It is easy to check that the kernel of $\phi$ as defined in the lemma has genus zero and the period $m_{3}$ is repeated $m$ times. The process can then be iterated.

Proof of Theorem 4.8 First observe that if $\mathbf{H}^{2} / \Gamma$ is an arithmetic integral homology 2-sphere, then $\Gamma=[\Gamma, \Gamma] \subset \Gamma_{\mathcal{O}}^{1}$ so that $\Gamma$ is derived from a quaternion algebra $A$ over $k=\mathbb{Q}(\operatorname{tr} \Gamma)$. But then $A$ must be unramified at all finite places since, otherwise, $\Gamma$ would have a non-trivial finite cyclic quotient (see e.g. [32]). This then implies, by Theorem 2.1 , that $n=[k: \mathbb{Q}]$ is odd. Now the co-area of $\Gamma_{\mathcal{O}}^{1}$ is $\frac{8 \pi \zeta_{k}(2) \Delta_{k}^{3 / 2}}{\left(4 \pi^{2}\right)^{n}}$ (see e.g. $\left.[27, \S 11.1]\right)$ so that the inequality at $(2)$ above shows that

$$
\Delta_{k}^{3 / 2} \leq \frac{16}{3}\left(4 \pi^{2}\right)^{n}
$$

The discriminant $\Delta_{k}$ of a totally real field $k$ has the lower bound given by Odlyzko [34] as $\Delta_{k} \geq 2.439 \times 10^{-4} \times(29.099)^{n}$. These inequalities show that $n \leq 10$. If $\Gamma_{\mathcal{O}}^{1}$ contains an element of order $m$, then $\mathbb{Q}(\cos \pi / m) \subset k$ so that $\phi(2 m) / 2 \mid n$. Those values of $m$ which satisfy this for some odd $n$ less than 10 are: 


\begin{tabular}{|c|c|c|c|c|c|c|c|}
\hline$m$ & 2 & 3 & 7 & 9 & 11 & 19 & 27 \\
\hline$\phi(2 m) / 2$ & 1 & 1 & 3 & 3 & 5 & 9 & 9 \\
\hline
\end{tabular}

The only signatures satisfying Lemma 4.9 are then the triangle groups $(0 ; 2,3,7 ; 0)$, $(0 ; 2,3,11 ; 0),(0 ; 2,3,19 ; 0),(0 ; 2,7,9 ; 0)$ and by Takeuchi $[41]$ only the first two are arithmetic.

Corollary 4.11. Every commensurability class which contains arithmetic Fuchsian groups of genus 0 contains infinitely many non-conjugate groups of genus 0 .

Proof: By Corollary 4.10, this result follows immediately for all commensurability classes except those containing the groups with signatures $(0 ; 2,3,7 ; 0)$ or $(0 ; 2,3,11 ; 0)$. However, the commensurability classes of these two groups contain groups of genus 0 which satisfy the conditions of Corollary 4.10 [29].

4.5. Proof of Theorem 1.5. Consider a cone point $a$ on $F$ of cone angle $2 \pi / \alpha$. The disc of radius $\rho$ centered at $a$ has circumference $2 \pi \sinh (\rho) / \alpha$ and exactly as in the proof of the $2 \pi$ theorem, for sufficiently large $\rho=\rho(\alpha)$, one can remove this disc and replace it by a smooth disc equipped with a metric of negative curvature. It follows that if we place a disc $D_{i}$ of radius $\rho\left(\alpha_{i}\right)$ about each cone point $a_{i}$ on the surface, this family of discs cannot be disjoint, else we could remove them all and replace each cone disc by a smooth disc equipped with a metric of negative curvature; thus obtaining a metric of negative curvature on a sphere or a torus which is impossible.

By shrinking two discs which do meet we see that we can find two loops on the surface, $\gamma_{1}$ and $\gamma_{2}$ which touch at one point, each representing finite order elements. By construction, $\gamma_{i}$ has length at most $2 \pi \sinh \left(\rho\left(\alpha_{i}\right)\right) / \alpha_{i}$, so that the loops $\gamma_{1} \cdot \gamma_{2}$ and $\gamma_{1} \cdot \gamma_{2}^{-1}$ both have length at most $2 \pi \sinh \left(\rho\left(\alpha_{1}\right)\right) / \alpha_{1}+2 \pi \sinh \left(\rho\left(\alpha_{2}\right)\right) / \alpha_{2}$.

If orientations are chosen so that, say, $\gamma_{1} \cdot \gamma_{2}$ is a simple loop, it cannot be nullhomotopic, else the surface has exactly two cone points and is either bad or spherical, both of which have been ruled out. If it represents a hyperbolic element we are done (it has length bounded above by a constant depending only on the order of the cone points), so the only possibility is that this element is elliptic and $F$ is a sphere with three cone points.

It follows that the elements $\gamma_{1}$ and $\gamma_{2}$ cannot both have order two, for this implies the cone angle at each cone point is $\pi$ and therefore the Euler characteristic of $F$ is positive, a contradiction. Suppose that $\gamma_{2}$ has order at least three; in the negatively curved triangle group

$$
\left\langle\gamma_{1}, \gamma_{2} \mid \gamma_{1}^{p}=\gamma_{2}^{q}=\left(\gamma_{1} \cdot \gamma_{2}\right)^{r}=1\right\rangle
$$

the element $\gamma_{1} \cdot \gamma_{2}^{-1}$ is hyperbolic and the proof is complete. 
Corollary 4.12. There is a constant $K$ (independent of genus), so that any hyperelliptic surface contains a geodesic of length $\leq K$.

From Corollary 4.5 and Theorem 1.5 we get.

Corollary 4.13. There is a constant $K$ such that, if $\Gamma$ is an arithmetic Fuchsian group of genus 0 , then $\mathbf{H}^{2} / \Gamma$ contains a geodesic of length $\leq K$.

Remark: Nathan Dunfield pointed out to us that a stronger version of Theorem 1.5 can be established (whereby the condition on the bound on the order of the torsion is removed).

Theorem 4.14. Let $F$ be a closed orientable hyperbolic 2-orbifold, with underlying topological surface a sphere or a torus.

Then there is a number $K$ (which is at most $4 \pi$ ) so that $F$ contains a geodesic of length at most $K$.

We will not include a proof here. The proof is similar to that of Theorem 1.5 with the added control given by the fact that (in the language of Theorem 1.5) it can be shown that as the radius $\rho\left(\alpha_{i}\right) \rightarrow \infty$ the circumference of the resulting circle is uniformly bounded.

\section{EXAMPLES}

Many examples of arithmetic Fuchsian groups of genus 0 are known. In this section, we give a complete enumeration of all maximal arithmetic Fuchsian groups of genus 0 which are defined over $\mathbb{Q}$. We also list some further examples for fields of larger degrees over $\mathbb{Q}$.

Recall that any Fuchsian group $\Gamma$ of finite co-area has a presentation of the form:

$$
\begin{aligned}
& <a_{1}, b_{1}, \ldots, a_{g}, b_{g}, x_{1}, \ldots, x_{r}, p_{1}, \ldots, p_{t} \mid x_{i}^{m_{i}}= \\
& =1(i=1, \ldots, r), \prod_{1}^{r} x_{i} \prod_{1}^{t} p_{j} \prod_{1}^{g}\left[a_{k}, b_{k}\right]=1>.
\end{aligned}
$$

The parameters appearing in the presentation - $\left(g ; m_{1}, \ldots, m_{r} ; t\right)$ - form the signature of the group $\Gamma$ and determine its isomorphism class. The integers $\left\{m_{1}, m_{2}, \ldots, m_{r}\right\}$ are the periods of the group. Its co-area is given by

$$
\text { Co }-\operatorname{area}(\Gamma)=2 \pi\left(2(g-1)+t+\sum_{i=1}^{r}\left(1-m_{i}^{-1}\right)\right) .
$$

Furthermore, $\mathbf{H}^{2} / \Gamma$ is non-cocompact if and only if $t \neq 0$. 
Now consider arithmetic Fuchsian groups defined by a quaternion algebra $B$ over a totally real number field $k$ as described in $\S 3$. In the cases where $\Gamma$ is non-cocompact, the maximal such groups are the normalizers $N\left(\Gamma_{0}(n)\right)$ of $\Gamma_{0}(n)$ for $n$ square-free as described in $\S 3.3$ and the group theoretic structure of these groups is described in $[17,24]$. From these descriptions, the values $n$ such that $N\left(\Gamma_{0}(n)\right)$ has genus 0 can readily be deduced as can the signature. These are tabulated in Table 1. (See also $[36,20]$ and Remarks 5.2 and 5.4 below).

So now let us suppose that $\Gamma$ is cocompact. All arithmetic triangle groups i.e. those with signatures $\left(0 ; m_{1}, m_{2}, m_{3} ; 0\right)$ have been determined in [41] and all those of signature $(0 ; 2,2,2, q ; 0)$ in $[42,28]$. Other cases where the signature has the form $\left(0 ; m_{1}, m_{2}, m_{3}, m_{4} ; 0\right)$ are considered in $[3,4]$. As already noted, there are only finitely many conjugacy classes of arithmetic Fuchsian groups of any given signature [42], but there are infinitely many conjugacy classes of arithmetic Fuchsian groups of genus 0 (Corollary 4.11). However, as given in Theorem 1.1, there are only finitely many conjugacy classes of maximal arithmetic Fuchsian groups of genus 0. As noted in $\S 3.2$, these groups have the form $\Gamma_{\mathcal{E}}^{+}=P\left(\rho\left(N(\mathcal{E})^{+}\right)\right)$ where $\mathcal{E}$ is an Eichler order of square-free level $S$ in the quaternion algebra $B$ and the decoration ' + ' indicates that all elements must have positive norm. The co-area of $\Gamma_{\mathcal{E}}^{+}$is given by the number theoretic data defining $B$ and $S[5]$ and the periods $\left\{m_{1}, m_{2}, \ldots, m_{r}\right\}$ can also be determined from this data $[25,26]$. Thus using (4), one can determine the genus.

5.1. Maximal arithmetic Fuchsian groups defined over $\mathbb{Q}$. Let $B$ be a quaternion algebra defined over $\mathbb{Q}$ which is indefinite, i.e. $B \otimes_{\mathbb{Q}} \mathbb{R} \cong M_{2}(\mathbb{R})$. The isomorphism class of $B$ is then determined by the finite set $\operatorname{Ram}_{f}(B)$, of even cardinality, of primes $p_{1}, p_{2}, \ldots, p_{2 m}$, these being the primes at which $B$ is ramified (see Theorem 2.1). Let $S$ be a finite set of finite primes disjoint from $\operatorname{Ram}_{f}(B)$ and let $\mathcal{E}$ be an Eichler order of square-free level $S$. All Eichler orders of level $S$ in these quaternion algebras over $\mathbb{Q}$ are conjugate in $B^{*}$. The maximal arithmetic Fuchsian groups in the commensurability class defined by $B$ are the groups $\Gamma_{\mathcal{E}}^{+}$as described in $\S 3.2$. Furthermore, [5],

$$
\text { Co }-\operatorname{area}\left(\Gamma_{\mathcal{E}}^{+}\right)=2 \pi\left[\frac{1}{6} \prod_{p \in \operatorname{Ram}_{f}(B)}\left(\frac{p-1}{2}\right) \prod_{q \in S}\left(\frac{q+1}{2}\right)\right] .
$$

For simplicity, we assume that $m \geq 1$, since the case $m=0$ corresponds to the non-cocompact groups. The formula given in [26] (see also [44]), from which one can determine the periods of any maximal arithmetic Fuchsian group becomes considerably simplified over $\mathbb{Q}$ and in the next subsection we deduce the periods of each $\Gamma_{\mathcal{E}}^{+}$in terms of the primes $p \in \operatorname{Ram}_{f}(B)$ and $q \in S$. 
5.2. Torsion in $\Gamma_{\mathcal{E}}^{+}$. Let $\operatorname{Ram}_{f}(B)=\left\{p_{1}, p_{2}, \ldots, p_{2 m}\right\}, S=\left\{q_{1}, q_{2}, \ldots, q_{s}\right\}$, and $D=\prod_{i=1}^{2 m} p_{i} \prod_{j=1}^{s} q_{j}$. Let $H(S)$ denote the subgroup of $\mathbb{Q}^{*}$ consisting of norms of elements in $N(\mathcal{E})^{+}$. Then $H(S)=\mathcal{D} \mathcal{S} \mathbb{Q}^{* 2}$ where $\mathcal{D}, \mathcal{S}$ are the subgroups of $\mathbb{Q}^{*}$ generated, respectively, by the $p_{i}$ and the $q_{j}$. The quotient group $\Gamma_{\mathcal{E}}^{+} / \Gamma_{\mathcal{E}}^{1} \cong$ $H(S) / \mathbb{Q}^{* 2}$ is an elementary abelian 2 -group of order $2^{2 m+s}$ whose elements are represented by the divisors $d$ of $D$ considered as elements of $\mathcal{D S}$.

Now suppose $\Gamma_{\mathcal{E}}^{+}$contains an element of order $r>2$. Then $r=3,4$ or 6 and there exists an embedding $\sigma: \mathbb{Q}\left(\mathrm{e}^{2 \pi i / r}\right) \rightarrow B$ such that $N(\mathcal{E})^{+}$contains a conjugate of $\sigma(u)$ where $u=1+\mathrm{e}^{2 \pi i / r}$ (see $[8,27]$ ). Conditions for the existence of such an embedding are given in Theorem 2.2 and are incorporated into the formula (6) below. Since $n(u) \in H(S)$ we must have $2+2 \cos 2 \pi / r \mid D$.

Now suppose that $\Gamma_{\mathcal{E}}^{+}$contains an element of order 2. Then there exists $\alpha \in$ $N(\mathcal{E})^{+}$such that $\alpha^{2} \in \mathbb{Q}^{*}$ and so $\alpha^{2}=-n(\alpha)$. Since $\alpha$ is only determined up to scalar multiples, we can take $n(\alpha)=d \mid D$. In this case, there exists an embedding $\sigma: \mathbb{Q}(\sqrt{-d}) \rightarrow B$ such that $N(\mathcal{E})^{+}$contains a conjugate of $\sigma(u)$ for $u=\sqrt{-d}$.

Thus, given $B$ and $S$, we define

$$
\begin{aligned}
& \mathcal{F}=\left\{u \in \mathbb{C} \mid u=1+\mathrm{e}^{2 \pi i / r}, r=3,4, \text { or } 6\right. \\
& \text { and } 2+2 \cos 2 \pi / r \mid D\} \cup\{u \in \mathbb{C} \mid u=\sqrt{-d} \text { where } d \mid D\} .
\end{aligned}
$$

For each $u \in \mathcal{F}$, let $L$ denote the quadratic imaginary field $\mathbb{Q}(u)$. The number of conjugacy classes of finite cyclic subgroups in $\Gamma_{\mathcal{E}}^{+}$will then be $\sum_{u \in \mathcal{F}} s(u)$ where $s(u)$ denotes the number of conjugacy classes of finite cyclic subgroups of $\Gamma_{\mathcal{E}}^{+}$corresponding to the element $u$. If $s(u) \neq 0$, there is an embedding $\sigma: L \rightarrow B$ and we let $\Omega$ denote the commutative $\mathbb{Z}$-order of $L$ which contains $u$ and is defined by $\sigma(L) \cap \mathcal{E}=\sigma(\Omega)$. The formula for $s(u)$ depends on $\Omega$, for which there are only two possibilities, either $\Omega=R_{L}$, the maximal order, or, in the cases where $d \equiv 3(\bmod 4), \Omega=\mathbb{Z}[\sqrt{-d}]$. We adopt the following notation (see $[13,44,26]$ )

$$
\left\{\frac{\Omega}{p}\right\}= \begin{cases}1 & \text { if } p=2 \text { and } \Omega \neq R_{L} \\ \left(\frac{L}{p}\right) & \text { otherwise }\end{cases}
$$

where, as usual, $\left(\frac{L}{p}\right)$ takes the values 1,0 or -1 according as $p$ splits, is ramified or is inert in the extension $L \mid \mathbb{Q}$. Then, adapting the formula in [26] to these cases, we have (cf. [44]):

$$
s(u)=\frac{h(L)}{2^{2 m+s-\eta(u)}} \sum_{\Omega \mid u \in \Omega} t(\Omega) \prod_{i=1}^{2 m}\left(1-\left\{\frac{\Omega}{p_{i}}\right\}\right) \prod_{j=1}^{s}\left(1+\left\{\frac{\Omega}{q_{j}}\right\}\right)
$$


where $h(L)$ is the class number of $L, \eta(u)=1$ unless $u=1+\mathrm{e}^{2 \pi i / 3}$ and $3 \not D$ or $u=\sqrt{-1}$ and $2 \not D$ in which cases $\eta(u)=0$, and

$$
t(\Omega)= \begin{cases}1 & \text { if } \Omega=R_{L} \\ \frac{2\left(1-\left(\frac{L}{2}\right) 2^{-1}\right)}{\left[R_{L}^{*}: B^{*}\right]} & \text { otherwise }\end{cases}
$$

It should be noted that necessary and sufficient conditions for $N(\mathcal{E})^{+}$to contain images of $u$ have been given in [8]. In these cases of groups defined over $\mathbb{Q}$, these conditions are incorporated into the definition of $\mathcal{F}$ and the formula for $s(u)$ at (6).

Theorem 5.1. Let $B$ be a quaternion algebra over $\mathbb{Q}$ with $\operatorname{Ram}_{f}(B)=\left\{p_{1}, p_{2}, \ldots\right.$, $\left.p_{2 m}\right\}, m \geq 1$, and let $S=\left\{q_{1}, q_{2}, \ldots, q_{s}\right\}$. Let $D=\prod_{i=1}^{2 m} p_{i} \prod_{j=1}^{s} q_{j}$ and let $\mathcal{E}$ be an Eichler order of level $S$. Then

(1) $\Gamma_{\mathcal{E}}^{+}$has elements of order 6 if and only if $3 \mid D,\left(\frac{\mathbb{Q}(\sqrt{-3})}{p_{i}}\right)=-1$ for all $p_{i}(\neq 3), i=1,2, \ldots, 2 m$ and $\left(\frac{\mathbb{Q}(\sqrt{-3})}{q_{j}}\right)=1$ for all $q_{j}(\neq 3), j=$ $1,2, \ldots, s$. There is then one conjugacy class of cyclic subgroups of order 6 in $\Gamma_{\mathcal{E}}^{+}$.

(2) $\Gamma_{\mathcal{E}}^{+}$has elements of order 4 if and only if $2 \mid D,\left(\frac{\mathbb{Q}(\sqrt{-1})}{p_{i}}\right)=-1$ for all $p_{i}(\neq 2), i=1,2, \ldots, 2 m$ and $\left(\frac{\mathbb{Q}(\sqrt{-1})}{q_{j}}\right)=1$ for all $q_{j}(\neq 2), j=$ $1,2, \ldots, s$. There is then one conjugacy class of cyclic subgroups of order 4 in $\Gamma_{\mathcal{E}}^{+}$.

(3) $\Gamma_{\mathcal{E}}^{+}$has elements of order 3 if and only if $\left(\frac{\mathbb{Q}(\sqrt{-3})}{p_{i}}\right)=-1$ for all $p_{i}(\neq 3)$, $i=1,2, \ldots, 2 m$ and $\left(\frac{\mathbb{Q}(\sqrt{-3})}{q_{j}}\right)=1$ for all $q_{j}(\neq 3), j=1,2, \ldots, s$. There is then one conjugacy class of cyclic subgroups of order 3 in $\Gamma_{\mathcal{E}}^{+}$.

Proof: Note that, in all cases, if $u \in \Omega$, then $\Omega=R_{L}$ so that there is only one term in the summation at $(6), t(\Omega)=1$ and $\left\{\frac{\Omega}{p}\right\}=\left(\frac{L}{p}\right)$. For $r=6, u \in \mathcal{F}$ if and only if $3 \mid D$. For $L=\mathbb{Q}(\sqrt{-3})$, then $s(u) \neq 0$ if and only if $\left(\frac{L}{p_{i}}\right) \neq 1$ for $i=1,2, \ldots, 2 m$ and $\left(\frac{L}{q_{j}}\right) \neq-1$ for $j=1,2, \ldots, s$. Since $h(L)=1$ and $\eta(u)=1$ the result for $r=6$ now follows. The same argument applies when $r=4, L=\mathbb{Q}(\sqrt{-1})$ and when $r=3, L=\mathbb{Q}(\sqrt{-3})$ and $3 \mid D$. If $r=3$ and $3 \not D$, we again have $s(u)=1$ as, in this case, $\eta(u)=0$. 
Remark 5.2. (1) Similar calculations to this were made in [14] and details appear in [1] for the groups $\Gamma_{\mathcal{E}}^{1}$ which correspond to Shimura curves.

(2) This result essentially appears in the guise of groups of units of orthogonal transformations of ternary quadratic forms over $\mathbb{Q}$ in [30].

(3) Re-interpreting the results for the non-cocompact case in [17, 24] shows that this result also holds true when $m=0$. Furthermore, the results in $[17,24]$ show that each $\Gamma_{\mathcal{E}}^{+}$contains just one conjugacy class of parabolic cyclic subgroups.

Now consider elements of order 2 in $\Gamma_{\mathcal{E}}^{+}$which correspond to $u=\sqrt{-d}, d \mid D$. For clarity, we replace $s(u)$ by $s(d)$. To simplify our statements, it is convenient to express our results in terms of $h^{2}(L)$ which is defined to be the order of $\mathcal{C}(L)^{2}$, the subgroup of the class group $\mathcal{C}(L)$ generated by the squares of elements in $\mathcal{C}(L)$. We also use $n(d)$ to denote the number of primes dividing $d$. Note that $h(L)=2^{n(d)-1} h^{2}(L)$ if $d \equiv 2,3(\bmod 4)$ and $h(L)=2^{n(d)} h^{2}(L)$ if $d \equiv 1(\bmod 4)$.

Theorem 5.3. Let $B$ be an indefinite quaternion algebra over $\mathbb{Q}$ with $\operatorname{Ram}_{f}(B)=$ $\left\{p_{1}, p_{2}, \ldots, p_{2 m}\right\}, m \geq 1$ and $S=\left\{q_{1}, q_{2}, \ldots, q_{s}\right\}$. Let $D=\prod_{i=1}^{2 m} p_{i} \prod_{j=1}^{s} q_{j}$ and let $\mathcal{E}$ be an Eichler order of level $S$. Let $s(d)$ denote the number of conjugacy classes of elements of order 2 in $\Gamma_{\mathcal{E}}^{+}$corresponding to $u=\sqrt{-d}$.

: a) Suppose $2 \nmid D$ and $d \equiv 1(\bmod 4)$.

$$
s(d)= \begin{cases}0 & \text { if }\left(\frac{L}{p_{i}}\right)=1 \text { for some } i \text { or }\left(\frac{L}{q_{j}}\right)=-1 \text { for some } j . \\ 1 & \text { if } d=1,\left(\frac{L}{p_{i}}\right) \neq 1 \text { for all } i \text { and }\left(\frac{L}{q_{j}}\right) \neq-1 \text { for all } j . \\ 2 h^{2}(L) \text { if } d \neq 1,\left(\frac{L}{p_{i}}\right) \neq 1 \text { for all } i \text { and }\left(\frac{L}{q_{j}}\right) \neq-1 \text { for all } j .\end{cases}
$$

: b) Suppose $2 \nmid D$ and $d \equiv 3(\bmod 4)$.

$$
s(d)= \begin{cases}0 \quad & \text { if }\left(\frac{L}{p_{i}}\right)=1 \text { for some } i \text { or }\left(\frac{L}{q_{j}}\right)=-1 \text { for some } j . \\ 2 \quad & \text { if } d=3,\left(\frac{L}{p_{i}}\right) \neq 1 \text { for all } i \text { and }\left(\frac{L}{q_{j}}\right) \neq-1 \text { for all } j . \\ 2 h^{2}(L) & \text { if } d \equiv 7(\bmod 8),\left(\frac{L}{p_{i}}\right) \neq 1 \text { for all } i \text { and }\left(\frac{L}{q_{j}}\right) \neq-1 \text { for all } j . \\ 4 h^{2}(L) & \text { if } d \neq 3, d \equiv 3(\bmod 8),\left(\frac{L}{p_{i}}\right) \neq 1 \\ & \text { for all } i \text { and }\left(\frac{L}{q_{j}}\right) \neq-1 \text { for all } j .\end{cases}
$$


: c) Suppose that $2 \in S$ and $d \equiv 1,2(\bmod 4)$ or that $2 \in \operatorname{Ram}_{f}(A)$.

$$
s(d)=\left\{\begin{array}{l}
0 \quad \text { if }\left(\frac{L}{p_{i}}\right)=1 \text { for some } i \text { and }\left(\frac{L}{q_{j}}\right)=-1 \text { for some } j . \\
h^{2}(L) \text { if }\left(\frac{L}{p_{i}}\right) \neq 1 \text { for all } i \text { and }\left(\frac{L}{q_{j}}\right) \neq-1 \text { for all } j .
\end{array}\right.
$$

: d) Suppose $2 \in S$ and $d \equiv 3(\bmod 4)$. then

$$
s(d)= \begin{cases}0 \quad & \text { if }\left(\frac{L}{p_{i}}\right)=1 \text { for some } i \text { and }\left(\frac{L}{q_{j}}\right)=-1 \text { for some } j, q_{j} \neq 2 . \\ 1 \quad & \text { if } d=3,\left(\frac{L}{p_{i}}\right) \neq 1 \text { for all } i \text { and }\left(\frac{L}{q_{j}}\right) \neq-1 \text { for all } j, q_{j} \neq 2 . \\ 2 h^{2}(L) & \text { if } d \equiv 7(\bmod 8),\left(\frac{L}{p_{i}}\right) \neq 1 \text { for all } i \text { and }\left(\frac{L}{q_{j}}\right) \neq-1 \\ & \text { for all } j, q_{j} \neq 2 . \\ 3 h^{2}(L) & \text { if } d \neq 3, d \equiv 3(\bmod 8),\left(\frac{L}{p_{i}}\right) \neq 1 \text { for all } i \text { and }\left(\frac{L}{q_{j}}\right) \neq-1 \\ \quad \text { for all } j, q_{j} \neq 2 .\end{cases}
$$

Proof: Consider the case where $d=1$. If $2 \mid D$, then $\eta(u)=1$, while, if $2 \nmid D$, $\eta(u)=0$. In both cases, exactly as in Theorem 5.1,if $s(1) \neq 0$, then $s(1)=1$.

So now assume that $d>1$ so that $\eta(u)=1$.

: a) Note that $\Omega=R_{L}$ in this case. Thus there is only one term in the summation at $(6), t(\Omega)=1$ and $\left\{\frac{\Omega}{p}\right\}=\left(\frac{L}{p}\right)$. The necessary and sufficient conditions for $s(d)=0$ are then immediate. Furthermore, $s(d)=h(L) 2^{2 m+s-n(d)} / 2^{2 m+s-1}=2 h^{2}(L)$.

: b) In this case there will be two terms in the summation at (6). As in a), the term corresponding to $\Omega=R_{L}$ yields $h(L) / 2^{n(d)-1}$. The term corresponding to $\Omega=\mathbb{Z}[\sqrt{-d}]$ yields $h(L) t(\Omega) / 2^{n(d)-1}$. Now $\left(\frac{L}{2}\right)= \pm 1$ according as $d \equiv 7,3(\bmod 8)$ and $\left[R_{L}^{*}: \Omega^{*}\right]=1$ unless $d=3$ when it is 3 . Thus $t(\Omega)=1$ if $d=3$ or $d \equiv 7(\bmod 8)$ and $t(\Omega)=3$ if $d \equiv 3(\bmod 8)$. The result follows.

: c) A similar argument applies in this case. Note that, when $2 \in \operatorname{Ram}_{f}(B)$ and $d \equiv 7(\bmod 8), s(d)=0$.

: d) In this case, if $\Omega=R_{L}$, then $\left(1+\left\{\frac{\Omega}{2}\right\}\right)=0,2$ according as $d \equiv$ $3,7(\bmod 8)$, while, for $\Omega=\mathbb{Z}[\sqrt{-d}],\left(1+\left\{\frac{\Omega}{2}\right\}\right)=2$ in both cases. The calculation now proceeds as before. 
Remark 5.4. As in Remark 5.2, this result remains true in the non-cocompact case i.e. when $m=0$ by the results of $[17,24]$.

5.3. Enumeration. From the results in $\S 5.2$, the signature of a maximal arithmetic Fuchsian group defind over $\mathbb{Q}$ has the form

$$
\left(g ; 6^{\left(n_{6}\right)}, 4^{\left(n_{4}\right)}, 3^{\left(n_{3}\right)}, 2^{\left(n_{2}\right)} ; n_{p}\right)
$$

where $a^{\left(n_{a}\right)}$ indicates that the period $a$ is repeated $n_{a}$ times. We have seen that $n_{6}, n_{4}, n_{3}, n_{p} \in\{0,1\}$ and, if $n_{6}=1$ then $n_{3}=0$. Also, in the notation of Theorem 5.3

$$
n_{2}=\left(\sum_{d \mid D} s(d)\right)-\left(n_{4}+n_{6}\right)
$$

Finally the genus $g$ can then be determined from the co-area formulas (4) and (5).

If the maximal group $\Gamma_{\mathcal{E}}^{+}$is to have genus 0 , then the bound obtained from Zograf's Theorem 4.4 and (2) show that

$$
\frac{1}{6} \prod_{p \in \operatorname{Ram}_{f}(A)}\left(\frac{p-1}{2}\right) \prod_{q \in S}\left(\frac{q+1}{2}\right) \leq \frac{64}{3}
$$

We can then determine all possibilities for $\operatorname{Ram}_{f}(A)$ and $S$ which satisfy this inequality, noting in particular that the number of primes in $\operatorname{Ram}_{f}(A) \cup S$ is no greater than 6 . For each such selection of primes we can determine the number of periods of each order and, in the non-cocompact case, the number of parabolic classes, from Theorems 5.1 and 5.3 (and the remarks following them). For the elements of order 2, we made extensive use of the lists provided by the Pari archive [48] of the structure of the class groups of $\mathbb{Q}(\sqrt{-d})$ for values of $d$ up to 40,000 . Finally the area formulas decide whether or not the genus is 0 . The complete list of all maximal arithmetic Fuchsian groups defined over $\mathbb{Q}$ of genus 0 is given in Tables $1,2 \mathrm{a}$ to $2 \mathrm{~d}$ and 3 . Note that the conjugacy class of such a

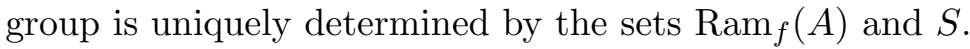

Remark 5.5. We note that the finiteness of the number of these groups over $\mathbb{Q}$ and, indeed, their enumeration can be exhibited independently of the inequality at (7) provided by Zograf. For the results of $\S 5$ show that the genus of $\Gamma_{\mathcal{E}}^{+}$where $\mathcal{E}$ is an Eichler order of level $S$ in the quaternion algebra $A$ satisfies 
(8)

$$
2 g \geq \frac{1}{6} \prod_{p \in \operatorname{Ram}_{f}(A)}\left(\frac{p-1}{2}\right) \prod_{q \in s}\left(\frac{q+1}{2}\right)-4 \sum_{d \mid D} \frac{h(\mathbb{Q}(\sqrt{-d}))}{2^{n(d)}}
$$

where, as in $\S 5, D$ is the product of the distinct primes in $\operatorname{Ram}_{f}(A) \cup S$ and $n(d)$ is the number of prime divisors of $d$. Now $h(\mathbb{Q}(\sqrt{-d})) \leq k \sqrt{d} \ln (d)$ for some fixed $k$. But then, the first term on the right hand side of (8) grows more quickly than the second so that, for large enough $D, g>0$. Since specific values can be obtained for the constant $k$ (see [23]), bounds on $D$ can be determined which allow an enumeration of the finite number of maximal arithmetic Fuchsian groups defined over $\mathbb{Q}$ of genus 0 as before.

\begin{tabular}{|ccc|}
\hline $\operatorname{Ram}_{f}(A)$ & $\mathrm{S}$ & Signature \\
\hline$\emptyset$ & $\emptyset$ & $(0 ; 2,3 ; 1)$ \\
$2,3,5$ & $\left(0 ; 2^{(5)} ; 1\right)$ \\
$2,3,7$ & $\left(0 ; 2^{(6)} ; 1\right)$ \\
$2,3,11$ & $\left(0 ; 2^{(8)} ; 1\right)$ \\
$2,3,13$ & $\left(0 ; 2^{(9)} ; 1\right)$ \\
$2,5,7$ & $\left(0 ; 2^{(8)} ; 1\right)$ \\
$2,5,11$ & $\left(0 ; 2^{(11)} ; 1\right)$ \\
$3,5,7$ & $\left(0 ; 2^{(10)} ; 1\right)$ \\
& \\
2,3 & $\left(0 ; 2^{(3)} ; 1\right)$ \\
2,5 & $\left(0 ; 4,2^{(2)} ; 1\right)$ \\
2,7 & $\left(0 ; 2^{(4)} ; 1\right)$ \\
2,11 & $\left(0 ; 2^{(5)} ; 1\right)$ \\
2,13 & $\left(0 ; 4,2^{(4)} ; 1\right)$ \\
2,17 & $\left(0 ; 4,2^{(5)} ; 1\right)$ \\
2,19 & $\left(0 ; 2^{(7)} ; 1\right)$ \\
2,23 & $\left(0 ; 2^{(8)} ; 1\right)$ \\
2,31 & $\left(0 ; 2^{(10)} ; 1\right)$ \\
2,47 & $\left(0 ; 2^{(14)} ; 1\right)$ \\
3,5 & $\left(0 ; 2^{(4)} ; 1\right)$ \\
3,7 & $\left(0 ; 6,2^{(3)} ; 1\right)$ \\
3,11 & $\left(0 ; 2^{(6)} ; 1\right)$ \\
3,13 & $\left(0 ; 6,2^{(5)} ; 1\right)$ \\
3,17 & $\left(0 ; 2^{(8)} ; 1\right)$ \\
\hline &
\end{tabular}

\begin{tabular}{|ccc|}
\hline $\operatorname{Ram}_{f}(A)$ & $\mathrm{S}$ & Signature \\
\hline$\emptyset$ & 3,23 & $\left(0 ; 2^{(10)} ; 1\right)$ \\
3,29 & $\left(0 ; 2^{(12)} ; 1\right)$ \\
3,47 & $\left(0 ; 2^{(18)} ; 1\right)$ \\
5,7 & $\left(0 ; 2^{(6)} ; 1\right)$ \\
5,11 & $\left(0 ; 2^{(8)} ; 1\right)$ \\
5,19 & $\left(0 ; 2^{(12)} ; 1\right)$ \\
7,17 & $\left(0 ; 2^{(14)} ; 1\right)$ \\
& \\
2 & $(0 ; 4,2 ; 1)$ \\
3 & $(0 ; 6,2 ; 1)$ \\
5 & $\left(0 ; 2^{(3)} ; 1\right)$ \\
7 & $\left(0 ; 3,2^{(2)} ; 1\right)$ \\
11 & $\left(0 ; 2^{(4)} ; 1\right)$ \\
13 & $\left(0 ; 3,2^{(3)} ; 1\right)$ \\
17 & $\left(0 ; 2^{(5)} ; 1\right)$ \\
19 & $\left(0 ; 3,2^{(4)} ; 1\right)$ \\
23 & $\left(0 ; 2^{(6)} ; 1\right)$ \\
29 & $\left(0 ; 2^{(7)} ; 1\right)$ \\
31 & $\left(0 ; 3,2^{(6)} ; 1\right)$ \\
41 & $\left(0 ; 2^{(9)} ; 1\right)$ \\
47 & $\left(0 ; 2^{(10)} ; 1\right)$ \\
59 & $\left(0 ; 2^{(12)} ; 1\right)$ \\
71 & $\left(0 ; 2^{(14)} ; 1\right)$ \\
\hline
\end{tabular}

Table 1. Non-cocompact groups. 


\begin{tabular}{|ccc|}
\hline $\operatorname{Ram}_{f}(A)$ & $\mathrm{S}$ & Signature \\
\hline 2,3 & $\emptyset$ & $(0 ; 2,4,6 ; 0)$ \\
5,7 & $\left(0 ; 2^{(6)} ; 0\right)$ \\
5,11 & $\left(0 ; 2^{(7)} ; 0\right)$ \\
5,13 & $\left(0 ; 4,2^{(6)} ; 0\right)$ \\
5,17 & $\left(0 ; 4,2^{(7)} ; 0\right)$ \\
5,19 & $\left(0 ; 2^{(9)} ; 0\right)$ \\
5,23 & $\left(0 ; 2^{(10)} ; 0\right)$ \\
5,29 & $\left(0 ; 4,2^{(10)} ; 0\right)$ \\
5,31 & $\left(0 ; 2^{(12)} ; 0\right)$ \\
5,47 & $\left(0 ; 2^{(16)} ; 0\right)$ \\
7,11 & $\left(0 ; 2^{(8)} ; 0\right)$ \\
7,13 & $\left(0 ; 6,2^{(7)} ; 0\right)$ \\
7,17 & $\left(0 ; 2^{(10} ; 0\right)$ \\
7,23 & $\left(0 ; 2^{(12)} ; 0\right)$ \\
7,29 & $\left(0 ; 2^{(14)} ; 0\right)$ \\
11,13 & $\left(0 ; 2^{(11)} ; 0\right)$ \\
& \\
5 & $\left(0 ; 4,2^{(3)} ; 0\right)$ \\
7 & $\left(0 ; 6,2^{(3)} ; 0\right)$ \\
11 & $\left(0 ; 2^{(5)} ; 0\right)$ \\
13 & $\left(0 ; 4,6,2^{(2)} ; 0\right)$ \\
17 & $\left(0 ; 4,2^{(4)} ; 0\right)$ \\
\hline
\end{tabular}

\begin{tabular}{|ccc|}
\hline $\operatorname{Ram}_{f}(A)$ & $\mathrm{S}$ & Signature \\
\hline 2,3 & 19 & $\left(0 ; 6,2^{(4)} ; 0\right)$ \\
& 23 & $\left(0 ; 2^{(6)} ; 0\right)$ \\
29 & $\left(0 ; 4,2^{(5)} ; 0\right)$ \\
31 & $\left(0 ; 6,2^{(5)} ; 0\right)$ \\
37 & $\left(0 ; 4,6,2^{(4)} ; 0\right)$ \\
41 & $\left(0 ; 4,2^{(6)} ; 0\right)$ \\
43 & $\left(0 ; 6,2^{(6)} ; 0\right)$ \\
47 & $\left(0 ; 2^{(8)} ; 0\right)$ \\
53 & $\left(0 ; 4,2^{(7)} ; 0\right)$ \\
59 & $\left(0 ; 2^{(9)} ; 0\right)$ \\
61 & $\left(0 ; 4,6,2^{(6)} ; 0\right)$ \\
67 & $\left(0 ; 6,2^{(8)} ; 0\right)$ \\
71 & $\left.\left(0 ;, 2^{(10}\right) ; 0\right)$ \\
73 & $\left(0 ; 4,6,2^{(7)} ; 0\right)$ \\
79 & $\left(0 ; 6,2^{(9)} ; 0\right)$ \\
83 & $\left(0 ; 2^{(11)} ; 0\right)$ \\
89 & $\left(0 ; 4,2^{(10)} ; 0\right)$ \\
97 & $\left(0 ; 4,6,2^{(9)} ; 0\right)$ \\
107 & $\left(0 ; 2^{(13)} ; 0\right)$ \\
109 & $\left(0 ; 4,6,2^{(10)} ; 0\right)$ \\
113 & $\left(0 ; 4,2^{(12)} ; 0\right)$ \\
137 & $\left(0 ; 4,2^{(14)} ; 0\right)$ \\
\hline
\end{tabular}

Table 2a. Cocompact groups commensurable with $(2,4,6)$ triangle group. 


\begin{tabular}{|c|c|c|}
\hline $\operatorname{Ram}_{f}(A)$ & $\mathrm{S}$ & Signature \\
\hline \multirow[t]{22}{*}{2,5} & $\emptyset$ & $\left(0 ; 3,2^{(3)} ; 0\right)$ \\
\hline & 3,7 & $\left(0 ; 6,2^{(5)} ; 0\right)$ \\
\hline & 3,11 & $\left(0 ; 2^{(8)} ; 0\right)$ \\
\hline & 3,13 & $\left(0 ; 6,2^{(7)} ; 0\right)$ \\
\hline & 3,17 & $\left(0 ; 2^{(10)} ; 0\right)$ \\
\hline & 3,19 & $\left(0 ; 6,2^{(9)} ; 0\right)$ \\
\hline & 3,23 & $\left(0 ; 2^{(12)} ; 0\right)$ \\
\hline & 3,31 & $\left(0 ; 6,2^{(13)} ; 0\right)$ \\
\hline & 7,11 & $\left(0 ; 2^{(12)} ; 0\right)$ \\
\hline & 3 & $\left(0 ; 6,2^{(3)} ; 0\right)$ \\
\hline & 7 & $\left(0 ; 3,2^{(4)} ; 0\right)$ \\
\hline & 11 & $\left(0 ; 2^{(6)} ; 0\right)$ \\
\hline & 13 & $\left(0 ; 3,2^{(5)} ; 0\right)$ \\
\hline & 17 & $\left(0 ; 2^{(7)} ; 0\right)$ \\
\hline & 19 & $\left(0 ; 3,2^{(6)} ; 0\right)$ \\
\hline & 23 & $\left(0 ; 2^{(8)} ; 0\right)$ \\
\hline & 29 & $\left(0 ; 2^{(9)} ; 0\right)$ \\
\hline & 31 & $\left(0 ; 3,2^{(8)} ; 0\right)$ \\
\hline & 37 & $\left(0 ; 3,2^{(9)} ; 0\right)$ \\
\hline & 41 & $\left(0 ; 2^{(11)} ; 0\right)$ \\
\hline & 53 & $\left(0 ; 2^{(13)} ; 0\right)$ \\
\hline & 61 & $\left(0 ; 3,2^{(13)} ; 0\right)$ \\
\hline \multirow[t]{13}{*}{2,7} & $\emptyset$ & $\left(0 ; 4,2^{(3)} ; 0\right)$ \\
\hline & 3,5 & $\left(0 ; 2^{(7)} ; 0\right)$ \\
\hline & 3,13 & $\left(0 ; 2^{(11)} ; 0\right)$ \\
\hline & 3,19 & $\left(0 ; 2^{(14)} ; 0\right)$ \\
\hline & 3 & $\left(0 ; 2^{(5)} ; 0\right)$ \\
\hline & 5 & $\left(0 ; 4,2^{(4)} ; 0\right)$ \\
\hline & 11 & $\left(0 ; 2^{(7)} ; 0\right)$ \\
\hline & 13 & $\left(0 ; 4,2^{(6)} ; 0\right)$ \\
\hline & 19 & $\left(0 ; 2^{(9)} ; 0\right)$ \\
\hline & 23 & $\left(0 ; 2^{(10)} ; 0\right)$ \\
\hline & 29 & $\left(0 ; 4,2^{(10)} ; 0\right)$ \\
\hline & 37 & $\left(0 ; 4,2^{(12)} ; 0\right)$ \\
\hline & 43 & $\left(0 ; 2^{(15)} ; 0\right)$ \\
\hline
\end{tabular}

\begin{tabular}{|c|c|c|}
\hline $\operatorname{Ram}_{f}(A)$ & $\mathrm{S}$ & Signature \\
\hline \multirow[t]{10}{*}{2,11} & $\emptyset$ & $\left(0 ; 3,4,2^{(2)} ; 0\right)$ \\
\hline & 3,5 & $\left(0 ; 2^{(9)} ; 0\right)$ \\
\hline & 3,7 & $\left(0 ; 6,2^{(9)} ; 0\right)$ \\
\hline & 5,7 & $\left(0 ; 2^{(14)} ; 0\right)$ \\
\hline & 3 & $\left(0 ; 6,2^{(4)} ; 0\right)$ \\
\hline & 5 & $\left(0 ; 4,2^{(5)} ; 0\right)$ \\
\hline & 7 & $\left(0 ; 3,2^{(6)} ; 0\right)$ \\
\hline & 13 & $\left(0 ; 3,4,2^{(7)} ; 0\right)$ \\
\hline & 17 & $\left(0 ; 4,2^{(10)} ; 0\right)$ \\
\hline & 19 & $\left(0 ; 3,2^{(11)} ; 0\right)$ \\
\hline \multirow[t]{7}{*}{2,13} & $\emptyset$ & $\left(0 ; 2^{(5)} ; 0\right)$ \\
\hline & 3,5 & $\left(0 ; 2^{(10)} ; 0\right)$ \\
\hline & 3,7 & $\left(0 ; 2^{(12)} ; 0\right)$ \\
\hline & 3 & $\left(0 ; 2^{(6)} ; 0\right)$ \\
\hline & 5 & $\left(0 ; 2^{(7)} ; 0\right)$ \\
\hline & 7 & $\left(0 ; 2^{(8)} ; 0\right)$ \\
\hline & 17 & $\left(0 ; 2^{(13)} ; 0\right)$ \\
\hline \multirow[t]{5}{*}{2,17} & $\emptyset$ & $\left(0 ; 3,2^{(4)} ; 0\right)$ \\
\hline & 3 & $\left(0 ; 6,2^{(5)} ; 0\right)$ \\
\hline & 5 & $\left(0 ; 2^{(8)} ; 0\right)$ \\
\hline & 7 & $\left(0 ; 3,2^{(8)} ; 0\right)$ \\
\hline & 11 & $\left(0 ; 2^{(12)} ; 0\right)$ \\
\hline \multirow[t]{4}{*}{2,19} & $\emptyset$ & $\left(0 ; 4,2^{(4)} ; 0\right)$ \\
\hline & 3 & $\left(0 ; 2^{(7)} ; 0\right)$ \\
\hline & 7 & $\left(0 ; 2^{(10)} ; 0\right)$ \\
\hline & 13 & $\left(0 ; 4,2^{(13)} ; 0\right)$ \\
\hline \multirow[t]{3}{*}{2,23} & $\emptyset$ & $\left(0 ; 3,4,2^{(3)} ; 0\right)$ \\
\hline & 3 & $\left(0 ; 6,2^{(6)} ; 0\right)$ \\
\hline & 5 & $\left(0 ; 4,2^{(8)} ; 0\right)$ \\
\hline \multirow[t]{3}{*}{2,29} & $\emptyset$ & $\left(0 ; 3,2^{(5)} ; 0\right)$ \\
\hline & 3 & $\left(0 ; 6,2^{(7)} ; 0\right)$ \\
\hline & 5 & $\left(0 ; 2^{(11)} ; 0\right)$ \\
\hline \multirow[t]{3}{*}{2,31} & $\emptyset$ & $\left(0 ; 4,2^{(5)} ; 0\right)$ \\
\hline & 3 & $\left(0 ; 2^{(9)} ; 0\right)$ \\
\hline & 7 & $\left(0 ; 2^{(14)} ; 0\right)$ \\
\hline
\end{tabular}

Table 2b. Cocompact groups with two ramified primes. 


\begin{tabular}{|c|c|c|}
\hline $\operatorname{Ram}_{f}(A)$ & $\mathrm{S}$ & Signature \\
\hline \multirow[t]{3}{*}{2,37} & $\emptyset$ & $\left(0 ; 2^{(7)} ; 0\right)$ \\
\hline & 3 & $\left(0 ; 2^{(10)} ; 0\right)$ \\
\hline & 5 & $\left(0 ; 2^{(13)} ; 0\right)$ \\
\hline \multirow[t]{2}{*}{2,41} & $\emptyset$ & $\left(0 ; 3,2^{(6)} ; 0\right)$ \\
\hline & 3 & $\left(0 ; 6,2^{(9)} ; 0\right)$ \\
\hline \multirow[t]{2}{*}{2,43} & $\emptyset$ & $\left(0 ; 4,2^{(6)} ; 0\right)$ \\
\hline & 3 & $\left(0 ; 2^{(11)} ; 0\right)$ \\
\hline 2,47 & $\emptyset$ & $\left(0 ; 3,4,2^{(5)} ; 0\right)$ \\
\hline 2,53 & $\emptyset$ & $\left(0 ; 3,2^{(7)} ; 0\right)$ \\
\hline 2,59 & $\emptyset$ & $\left(0 ; 3,4,2^{(6)} ; 0\right)$ \\
\hline 2,61 & $\emptyset$ & $\left(0 ; 2^{(9)} ; 0\right)$ \\
\hline \multirow[t]{2}{*}{2,67} & $\emptyset$ & $\left(0 ; 4,2^{(8)} ; 0\right)$ \\
\hline & 3 & $\left(0 ; 2^{(15)} ; 0\right)$ \\
\hline 2,73 & $\emptyset$ & $\left(0 ; 2^{(10)} ; 0\right)$ \\
\hline 2,83 & $\emptyset$ & $\left(0 ; 3,4,2^{(8)} ; 0\right)$ \\
\hline 2,89 & $\emptyset$ & $\left(0 ; 3,2^{(10)} ; 0\right)$ \\
\hline 2,97 & $\emptyset$ & $\left(0 ; 2^{(12)} ; 0\right)$ \\
\hline 2,101 & $\emptyset$ & $\left(0 ; 3,2^{(11)} ; 0\right)$ \\
\hline 2,103 & $\emptyset$ & $\left(0 ; 4,2^{(11)} ; 0\right)$ \\
\hline 2,157 & $\emptyset$ & $\left(0 ; 2^{(17)} ; 0\right)$ \\
\hline \multirow[t]{13}{*}{3,5} & $\emptyset$ & $\left(0 ; 6,2^{(3)} ; 0\right)$ \\
\hline & 2,7 & $\left(0 ; 2^{(8)} ; 0\right)$ \\
\hline & 2,11 & $\left(0 ; 2^{(10)} ; 0\right)$ \\
\hline & 2,13 & $\left(0 ; 2^{(11)} ; 0\right)$ \\
\hline & 2,17 & $\left(0 ; 2^{(13)} ; 0\right)$ \\
\hline & 2 & $\left(0 ; 2^{(5)} ; 0\right)$ \\
\hline & 7 & $\left(0 ; 6,2^{(5)} ; 0\right)$ \\
\hline & 11 & $\left(0 ; 2^{(8)} ; 0\right)$ \\
\hline & 13 & $\left(0 ; 6,2^{(7)} ; 0\right)$ \\
\hline & 17 & $\left(0 ; 2^{(10)} ; 0\right)$ \\
\hline & 19 & $\left(0 ; 6,2^{(9)} ; 0\right)$ \\
\hline & 29 & $\left(0 ; 2^{(14)} ; 0\right)$ \\
\hline & 41 & $\left(0 ; 2^{(18)} ; 0\right)$ \\
\hline
\end{tabular}

\begin{tabular}{|c|c|c|}
\hline $\operatorname{Ram}_{f}(A)$ & $\mathrm{S}$ & Signature \\
\hline \multirow[t]{9}{*}{3,7} & $\emptyset$ & $\left(0 ; 2^{(5)} ; 0\right)$ \\
\hline & 2,5 & $\left(0 ; 4,2^{(7)} ; 0\right)$ \\
\hline & 2,11 & $\left(0 ; 2^{(13)} ; 0\right)$ \\
\hline & 2,13 & $\left(0 ; 4,2^{(13)} ; 0\right)$ \\
\hline & 2 & $\left(0 ; 4,2^{(4)} ; 0\right)$ \\
\hline & 5 & $\left(0 ; 2^{(7)} ; 0\right)$ \\
\hline & 11 & $\left(0 ; 2^{(10)} ; 0\right)$ \\
\hline & 13 & $\left(0 ; 2^{(11)} ; 0\right)$ \\
\hline & 19 & $\left(0 ; 2^{(14)} ; 0\right)$ \\
\hline \multirow[t]{6}{*}{3,11} & $\emptyset$ & $\left(0 ; 6,2^{(4)} ; 0\right)$ \\
\hline & 2,5 & $\left(0 ; 4,2^{(10)} ; 0\right)$ \\
\hline & 2,7 & $\left(0 ; 2^{(14)} ; 0\right)$ \\
\hline & 2 & $\left(0 ; 4,2^{(5)} ; 0\right)$ \\
\hline & 5 & $\left(0 ; 2^{(9)} ; 0\right)$ \\
\hline & 7 & $\left(0 ; 6,2^{(9)} ; 0\right)$ \\
\hline \multirow[t]{5}{*}{3,13} & $\emptyset$ & $\left(0 ; 2^{(6)} ; 0\right)$ \\
\hline & 2,5 & $\left(0 ; 2^{(13)} ; 0\right)$ \\
\hline & 2 & $\left(0 ; 2^{(7)} ; 0\right)$ \\
\hline & 5 & $\left(0 ; 2^{(10)} ; 0\right)$ \\
\hline & 7 & $\left(0 ; 2^{(12)} ; 0\right)$ \\
\hline \multirow[t]{4}{*}{3,17} & $\emptyset$ & $\left(0 ; 6,2^{(5)} ; 0\right)$ \\
\hline & 2,5 & $\left(0 ; 2^{(16)} ; 0\right)$ \\
\hline & 2 & $\left(0 ; 2^{(8)} ; 0\right)$ \\
\hline & 5 & $\left(0 ; 2^{(12)} ; 0\right)$ \\
\hline \multirow[t]{3}{*}{3,19} & $\emptyset$ & $\left(0 ; 2^{(7)} ; 0\right)$ \\
\hline & 2 & $\left(0 ; 4,2^{(7)} ; 0\right)$ \\
\hline & 7 & $\left(0 ; 2^{(16)} ; 0\right)$ \\
\hline \multirow[t]{2}{*}{3,23} & $\emptyset$ & $\left(0 ; 6,2^{(6)} ; 0\right)$ \\
\hline & 2 & $\left(0 ; 4,2^{(8)} ; 0\right)$ \\
\hline \multirow[t]{2}{*}{3,29} & $\emptyset$ & $\left(0 ; 6,2^{(7)} ; 0\right)$ \\
\hline & 2 & $\left(0 ; 2^{(11)} ; 0\right)$ \\
\hline
\end{tabular}

Table 2c. Cocompact groups with two ramified primes. 


\begin{tabular}{|ccc|}
\hline $\operatorname{Ram}_{f}(A)$ & $\mathrm{S}$ & Signature \\
\hline 3,31 & $\emptyset$ & $\left(0 ; 2^{(9)} ; 0\right)$ \\
& 2 & $\left(0 ; 4,2^{(10)} ; 0\right)$ \\
3,37 & $\emptyset$ & $\left(0 ; 2^{(10)} ; 0\right)$ \\
& 2 & $\left(0 ; 2^{(13)} ; 0\right)$ \\
3,43 & $\emptyset$ & $\left(0 ; 2^{(11)} ; 0\right)$ \\
3,53 & $\emptyset$ & $\left(0 ; 6,2^{(11)} ; 0\right)$ \\
& 2 & $\left(0 ; 2^{(17)} ; 0\right)$ \\
3,61 & $\emptyset$ & $\left(0 ; 2^{(14)} ; 0\right)$ \\
3,79 & $\emptyset$ & $\left(0 ; 2^{(17)} ; 0\right)$ \\
5,7 & $\emptyset$ & $\left(0 ; 2^{(6)} ; 0\right)$ \\
& 2,3 & $\left(0 ; 2^{(10)} ; 0\right)$ \\
& 2 & $\left(0 ; 2^{(7)} ; 0\right)$ \\
& 3 & $\left(0 ; 2^{(8)} ; 0\right)$ \\
& 13 & $\left(0 ; 2^{(18)} ; 0\right)$ \\
5,11 & $\emptyset$ & $\left(0 ; 3,2^{(6)} ; 0\right)$ \\
& 2 & $\left(0 ; 2^{(9)} ; 0\right)$ \\
& 3 & $\left(0 ; 6,2^{(9)} ; 0\right)$ \\
5,13 & $\emptyset$ & $\left(0 ; 2^{(8)} ; 0\right)$ \\
& 3 & $\left(0 ; 2^{(12)} ; 0\right)$ \\
5,17 & $\emptyset$ & $\left(0 ; 3,2^{(8)} ; 0\right)$ \\
& 3 & $\left(0 ; 6,2^{(13)} ; 0\right)$ \\
5,19 & $\emptyset$ & $\left(0 ; 2^{(10)} ; 0\right)$ \\
& 2 & $\left(0 ; 2^{(13)} ; 0\right)$ \\
& 3 & $\left(0 ; 2^{(16)}: 0\right)$ \\
5,23 & $\emptyset$ & $\left(0 ; 3,2^{(10)} ; 0\right)$ \\
& 2 & $\left(0 ; 2^{(15)} ; 0\right)$ \\
5,43 & $\emptyset$ & $\left(0 ; 2^{(18)} ; 0\right)$ \\
7,11 & $\emptyset$ & $\left(0 ; 2^{(9)} ; 0\right)$ \\
& 2 & $\left(0 ; 4,2^{(10} ; 0\right)$ \\
7,17 & $\emptyset_{1}$ & $\left(0 ; 2^{(14)} ; 0\right)$ \\
& 2 & $\left(0 ; 2^{(12)} ; 0\right)$ \\
& 2 & $\left(0 ; 2^{(16)} ; 0\right)$ \\
& $\emptyset$ & $\left(0 ; 2^{(15)} ; 0\right)$ \\
& $\emptyset$ & $\left(0 ; 2^{(14)} ; 0\right)$ \\
& 2 & $\left(0 ; 2^{(19)} ; 0\right)$ \\
\hline & & \\
& & \\
5,13 &
\end{tabular}

Table 2d. Cocompact groups with two ramified primes. 


\begin{tabular}{|ccc|}
\hline $\operatorname{Ram}_{f}(A)$ & $\mathrm{S}$ & Signature \\
\hline $2,3,5,7$ & $\emptyset$ & $\left(0 ; 2^{(5)} ; 0\right)$ \\
& 11 & $\left(0 ; 2^{(10)} ; 0\right)$ \\
& 13 & $\left(0 ; 2^{(11)} ; 0\right)$ \\
& 17 & $\left(0 ; 2^{(13)} ; 0\right)$ \\
& 19 & $\left(0 ; 2^{(14)} ; 0\right)$ \\
$2,3,5,11$ & $\emptyset$ & $\left(0 ; 6,2^{(4)} ; 0\right)$ \\
& 7 & $\left(0 ; 6,2^{(9)} ; 0\right)$ \\
& 13 & $\left(0 ; 6,2^{(14)} ; 0\right)$ \\
$2,3,5,13$ & $\emptyset$ & $\left(0 ; 2^{(6)} ; 0\right)$ \\
& 7 & $\left(0 ; 2^{(12)} ; 0\right)$ \\
$2,3,5,17$ & $\emptyset$ & $\left(0 ; 6,2^{(5)} ; 0\right)$ \\
& 7 & $\left(0 ; 6,2^{(13)} ; 0\right)$ \\
$2,35,19$ & $\emptyset$ & $\left(0 ; 2^{(7)} ; 0\right)$ \\
& 7 & $\left(0 ; 2^{(16)} ; 0\right)$ \\
$2,35,23$ & $\emptyset$ & $\left(0 ; 6,2^{(6)} ; 0\right)$ \\
$2,3,5,29$ & $\emptyset$ & $\left(0 ; 6,2^{(7)} ; 0\right)$ \\
$2,3,5,31$ & $\emptyset$ & $\left(0 ; 2^{(9)} ; 0\right)$ \\
$2,3,5,37$ & $\emptyset$ & $\left(0 ; 2^{(10)} ; 0\right)$ \\
$2,3,5,41$ & $\emptyset$ & $\left(0 ; 6,2^{(9)} ; 0\right)$ \\
$2,3,5,43$ & $\emptyset$ & $\left(0 ; 2^{(11)} ; 0\right)$ \\
$2,3,5,47$ & $\emptyset$ & $\left(0 ; 6,2^{(10)} ; 0\right)$ \\
$2,3,5,53$ & $\emptyset$ & $\left(0 ; 6,2^{(11)} ; 0\right)$ \\
$2,3,5,59$ & $\emptyset$ & $\left(0 ; 6,2^{(12)} ; 0\right)$ \\
$2,3,5,67$ & $\emptyset$ & $\left(0 ; 2^{(15)} ; 0\right)$ \\
\hline
\end{tabular}

\begin{tabular}{|ccc|}
\hline $\operatorname{Ram}_{f}(A)$ & $\mathrm{S}$ & Signature \\
\hline $2,3,5,71$ & $\emptyset$ & $\left(0 ; 6,2^{(14)} ; 0\right)$ \\
$2,3,7,11$ & $\emptyset$ & $\left(0 ; 4,2^{(5)} ; 0\right)$ \\
& 5 & $\left(0 ; 4,2^{(10)} ; 0\right)$ \\
$2,3,7,13$ & $\emptyset$ & $\left(0 ; 2^{(7)} ; 0\right)$ \\
& 5 & $\left(0 ; 2^{(13)} ; 0\right)$ \\
$2,3,7,17$ & $\emptyset$ & $\left(0 ; 2^{(8)} ; 0\right)$ \\
$2,3,7,19$ & $\emptyset$ & $\left(0 ; 4,2^{(7)} ; 0\right)$ \\
$2,37,23$ & $\emptyset$ & $\left(0 ; 4,2^{(8)} ; 0\right)$ \\
$2,37,29$ & $\emptyset$ & $\left(0 ; 2^{(11)} ; 0\right)$ \\
$2,3,7,31$ & $\emptyset$ & $\left(0 ; 4,2^{(10)} ; 0\right)$ \\
$2,3,7,41$ & $\emptyset$ & $\left(0 ; 2^{(14)} ; 0\right)$ \\
$2,3,7,47$ & $\emptyset$ & $\left(0 ; 4,2^{(14)} ; 0\right)$ \\
$2,3,11,13$ & $\emptyset$ & $\left(0 ; 2^{(9)} ; 0\right)$ \\
$2,3,11,17$ & $\emptyset$ & $\left(0 ; 6,2^{(9)} ; 0\right)$ \\
$2,3,11,19$ & $\emptyset$ & $\left(0 ; 4,2^{(10)} ; 0\right)$ \\
$2,3,11,29$ & $\emptyset$ & $\left(0 ; 6,2^{(14)} ; 0\right)$ \\
$2,3,13,17$ & $\emptyset$ & $\left(0 ; 2^{(12)}: 0\right)$ \\
$2,3,13,23$ & $\emptyset$ & $\left(0 ; 2^{(15)} ; 0\right)$ \\
$2,3,17,19$ & $\emptyset$ & $\left(0 ; 2^{(16)} ; 0\right)$ \\
$2,5,7,11$ & $\emptyset$ & $\left(0 ; 2^{(9)} ; 0\right)$ \\
& 3 & $\left(0 ; 2^{(14)} ; 0\right)$ \\
$2,5,7,13$ & $\emptyset$ & $\left(0 ; 2^{(10)} ; 0\right)$ \\
$2,5,7,17$ & $\emptyset$ & $\left(0 ; 2^{(12)}: 0\right)$ \\
& & \\
\hline
\end{tabular}

Table 3. Cocompact groups with four ramified primes.

5.4. Further examples. For several signatures, all conjugacy classes of arithmetic Fuchsian groups of that signature have been listed. In particular, for cocompact groups of genus 0 and $r=3$ this was done by Takeuchi, [41], for $(0 ; 2,2,2, q ; 0)$ see $[42,28]$ and [3], and for an extensive list of genus 0 and $r=4$ see [4]. For quadratic fields, [19] provides lists, and we incorporate some of these in our table. We have done extensive calculations in search of examples of genus 0 , and have found many up through degree 7 . Table 4 displays part of these calculations. The columns of the table show the degree of the field $k$ over $\mathbb{Q}$, the discriminant of the field $k$ (which in these cases uniquely determines it), the finite places at which the associated quaternion algebra is ramified, and the signature of the maximal group $\Gamma_{\mathcal{O}}^{+}$, where $\mathcal{O}$ is a maximal order of $B$ (using the same conventions as in Tables 1 to 3 ). These groups are of minimal covolume in their commensurability class. 
In $\S 5.2$ we adapted the formula given in [26] to give a more amenable form for calculating the periods of maximal arithmetic Fuchsian groups defined over $\mathbb{Q}$. Here we again give simplified versions of the formula in $[25]$ which determines the periods in $\Gamma_{\mathcal{O}}^{+}$where $\mathcal{O}$ is a maximal order in some quaternion algebra $B$ over the field $k$. For this we introduce some notation, as we did at the start of $\$ 5.2$ (see $[5,25]$ for details). Let $\mathcal{D}$ denote the subgroup of the group $I_{k}$ of fractional ideals of $k$ generated by all $\mathcal{P} \in \operatorname{Ram}_{f}(B)$. Then $\Gamma_{\mathcal{O}}^{+} / \Gamma_{\mathcal{O}^{1}} \cong H / k^{* 2}$ where $H=\left\{x \in k^{* 2} \mid x R_{k} \in \mathcal{D} I_{k}^{2}\right\}$. In the cases where $k$ has class number 1 , $\Gamma_{\mathcal{O}}^{+} / \Gamma_{\mathcal{O}^{1}} \cong R_{f,+}^{*} / R_{f}^{* 2}$ where $R_{f}$ is the ring of elements in $k$ which are integral at all finite places not in $\operatorname{Ram}_{f}(B)$. Let $\mathcal{R}$ be a set of coset representatives of $H$ modulo $k^{* 2}$ (or $R_{f,+}^{*}$ modulo $R_{f}^{* 2}$ ).

Now $\Gamma_{\mathcal{O}}^{+}$will contain elements of order $m>2$ provided $m \in \mathcal{I}$ which consists of all $m$ such that (i) $2 \cos 2 \pi / m \in k$, (ii) no prime $\mathcal{P} \in \operatorname{Ram}_{f}(B)$ splits in $k\left(\mathrm{e}^{2 \pi i / m}\right) \mid$ $k$ and (iii) $2+2 \cos 2 \pi / m \in H$. Also $\Gamma_{\mathcal{O}}^{+}$will contain elements of order 2 for each $n \in \mathcal{R}(B)$ where $\mathcal{R}(B)=\left\{n \in \mathcal{R} \mid\right.$ no prime in $\operatorname{Ram}_{f}(B)$ splits in $\left.k(\sqrt{-n}) \mid k\right\}$. For $m \in \mathcal{I}$ or $n \in \mathcal{R}(B)$, let $u=1+\mathrm{e}^{2 \pi i / m}$ or $\sqrt{-n}$ respectively. The number of periods in $\Gamma_{\mathcal{O}}^{+}$corresponding to $m$ or $n$ is given by the number $s(u)$ for which a formula is given below under special circumstances which apply to many of the examples on Table 4.

For any such $u$ as just described, let $L$ denote the totaly imaginary quadratic extension $k(u)$ of $k$. If there exists $t \in k$ such that $\{1, t u\}$ is a relative integral basis of $L \mid k$, then

$$
s(u)=\frac{h(L)}{h} \frac{1}{\left[R_{k,+}^{*}: R_{k}^{* 2}\right]} \frac{1}{\left[H: N_{L \mid k}\left(R_{L}^{\prime}\right) R_{k,+}^{*} k^{* 2}\right]} \prod_{\mathcal{P} \mid \Delta(B)}\left(1-\left(\frac{L}{\mathcal{P}}\right)\right)
$$

where $h(L), h$ are the class numbers of $L, k$ respectively, $R_{k,+}^{*}$ is the group of totally positive units in $R_{k}$ and

$$
R_{L}^{\prime}=\left\{\alpha \in R_{L} \mid N_{L \mid k}(\alpha) \in H \text { and } \operatorname{Tr}_{L \mid k}^{2}(\alpha) \in N_{L \mid k}(\alpha) R_{k}\right\}
$$

We refer to the factor $\left[H: N_{L \mid k}\left(R_{L}^{\prime}\right) R_{k,+}^{*} k^{* 2}\right]$ as the H-index. When the class number of $k$ is 1 , as noted above, $H$ can be replaced by $R_{f,+}^{*}$ and the H-index becomes $\left[R_{f,+}^{*}: N_{L \mid k}\left(R_{L}^{\prime}\right) R_{k,+}^{*} R_{f}^{* 2}\right]$. In addition, if $\operatorname{Ram}_{f}(B)=\emptyset$, then $R_{f,+}^{*}=$ $R_{k,+}^{*}$ and the H-index is 1. The formula for $s(u)$ given in [25] and at (9) enables one to determine the periods of the maximal groups $\Gamma_{\mathcal{O}}^{+}$and the co-area is also given [5] in terms of the arithmetic data by

$$
\text { Co }- \text { area } \Gamma_{\mathcal{O}}^{+}=\frac{8 \pi \zeta_{k}(2) d_{k}^{3 / 2} \prod_{\mathcal{P} \mid \Delta(B)}(N \mathcal{P}-1)}{\left(4 \pi^{2}\right)^{[k: \mathbb{Q}]}\left[H: k^{* 2}\right]} .
$$




\begin{tabular}{|c|c|c|c|}
\hline Degree & Discriminant & $\operatorname{Ram}_{f} B$ & Signature of $\Gamma_{\mathcal{O}}^{+}$ \\
\hline 1 & 1 & $\emptyset$ & $(0 ; 2,3 ; 1)$ \\
\hline 1 & 1 & 2,3 & $(0 ; 2,4,6 ; 0)$ \\
\hline 1 & 1 & 2,5 & $\left(0 ; 2^{(3)}, 3 ; 0\right)$ \\
\hline 1 & 1 & 2,11 & $\left(0 ; 2^{(2)}, 3,4 ; 0\right)$ \\
\hline 2 & 5 & $\wp_{2}$ & $(0 ; 2,4,5 ; 0)$ \\
\hline 2 & 5 & $\wp_{3}$ & $(0 ; 2,5,6 ; 0)$ \\
\hline 2 & 5 & $\wp_{5}$ & $(0 ; 2,3,10 ; 0)$ \\
\hline 2 & 5 & $\wp_{11}$ & $\left(0 ; 2^{(3)}, 3 ; 0\right)$ \\
\hline 2 & 5 & $\wp_{59}$ & $\left(0 ; 2^{(3)}, 3,5 ; 0\right)$ \\
\hline 2 & 8 & $\wp_{2}$ & $(0 ; 2,3,8 ; 0)$ \\
\hline 2 & 12 & $\wp_{11}$ & $(0 ; 2,3,4,12 ; 0)$ \\
\hline 2 & 61 & $\wp_{3}$ & $\left(0 ; 2^{(5)}, 3^{(2)} ; 0\right)$ \\
\hline 2 & 120 & $\wp_{2}$ & $\left(0 ; 2,3^{(2)}, 4,6 ; 0\right)$ \\
\hline 3 & 49 & $\emptyset$ & $(0 ; 2,3,7 ; 0)$ \\
\hline 3 & 81 & $\emptyset$ & $(0 ; 2,3,9 ; 0)$ \\
\hline 3 & 81 & $\wp_{2}, \wp_{3}$ & $\left(0 ; 2^{(3)}, 9 ; 0\right)$ \\
\hline 3 & 148 & $\emptyset$ & $\left(0 ; 2^{(3)}, 3 ; 0\right)$ \\
\hline 3 & 148 & $\wp_{2}, \wp_{5}$ & $\left(0 ; 2^{(3)}, 3 ; 0\right)$ \\
\hline 3 & 169 & $\emptyset$ & $\left(0 ; 2^{(3)}, 3 ; 0\right)$ \\
\hline 3 & 564 & $\emptyset$ & $\left(0 ; 2^{(3)}, 3^{(3)} ; 0\right)$ \\
\hline 3 & 761 & $\emptyset$ & $\left(0 ; 2^{(3)}, 3^{(2)} ; 0\right)$ \\
\hline 3 & 785 & $\emptyset$ & $\left(0 ; 2^{(5)}, 3^{(2)} ; 0\right)$ \\
\hline 3 & 985 & $\emptyset$ & $\left(0 ; 2^{(5)}, 3 ; 0\right)$ \\
\hline 4 & 725 & $\wp 11$ & $\left(0 ; 2^{(3)}, 3 ; 0\right)$ \\
\hline 4 & 725 & $\wp_{19}$ & $\left(0 ; 2^{(3)}, 5 ; 0\right)$ \\
\hline 4 & 1125 & $\wp_{3}$ & $(0 ; 2,5,30 ; 0)$ \\
\hline 4 & 1125 & $\wp_{5}$ & $(0 ; 2,3,30 ; 0)$ \\
\hline 4 & 1600 & $\wp_{2}$ & $(0 ; 2,5,8 ; 0)$ \\
\hline 4 & 1957 & $\wp 3$ & $\left(0 ; 2^{(3)}, 3 ; 0\right)$ \\
\hline 4 & 2000 & $\wp_{2}$ & $(0 ; 2,5,20 ; 0)$ \\
\hline 4 & 2000 & $\wp_{5}$ & $\left(0 ; 2^{(3)}, 3 ; 0\right)$ \\
\hline 4 & 3600 & $\wp_{2}$ & $\left(0 ; 2^{(3)}, 5 ; 0\right)$ \\
\hline 4 & 9301 & $\wp_{3}$ & $\left(0 ; 2^{(5)}, 3^{(2)} ; 0\right)$ \\
\hline 4 & 10273 & $\wp_{2}$ & $\left(0 ; 2^{(4)}, 3^{(2)} ; 0\right)$ \\
\hline 4 & 10889 & $\wp_{2}$ & $\left(0 ; 2^{(4)}, 3^{(2)} ; 0\right)$ \\
\hline 5 & 14641 & $\emptyset$ & $(0 ; 2,3,11 ; 0)$ \\
\hline 5 & 24217 & $\emptyset$ & $\left(0 ; 2^{(3)}, 3 ; 0\right)$ \\
\hline 5 & 36497 & $\emptyset$ & $\left(0 ; 2^{(3)}, 3 ; 0\right)$ \\
\hline 5 & 38569 & $\emptyset$ & $\left(0 ; 2^{(3)}, 3 ; 0\right)$ \\
\hline 5 & 65657 & $\emptyset$ & $\left(0 ; 2^{(3)}, 3^{(2)} ; 0\right)$ \\
\hline 5 & 70601 & $\emptyset$ & $\left(0 ; 2^{(3)}, 3^{(2)} ; 0\right)$ \\
\hline 6 & 300125 & $\wp_{2}$ & $\left(0 ; 2^{(9)}, 4^{(3)}, 5 ; 0\right)$ \\
\hline 7 & 20134393 & $\emptyset$ & $\left(0 ; 2^{(5)}, 3^{(3)} ; 0\right)$ \\
\hline 7 & 41153941 & $\emptyset$ & $\left(0 ; 2^{(10)}, 3^{(7)} ; 0\right)$ \\
\hline
\end{tabular}

Table 4. Some genus 0 examples from low degree fields 
In all the examples that follow extensive use has been made of Pari [9] to determine the arithmetic information required to enumerate the periods and evaluate the co-area from the formula at (10).

Example 1: $[k: \mathbb{Q}]=3, d_{k}=985, \operatorname{Ram}_{f}(B)=\emptyset$.

Here $k=\mathbb{Q}(t)$ where $t$ satisfies $t^{3}+t^{2}-6 t-1=0$ and $h_{k}=1$. The fundamental units are $t, t-2$ and $R_{f,+}^{*}=R_{k,+}^{*}$ has order 2 modulo $R_{k}^{* 2}$ and is generated by $t(t-2)$. Estimating $\zeta_{k}(2)$ using Pari, we obtain the co-area of $\Gamma_{\mathcal{O}}^{+}$is $14 \pi / 6$. Since $\operatorname{Ram}_{f}(B)=\emptyset$, we see that $2,3 \notin H$ so there are no elements of order 4 or 6 and that there will be elements of order 3 and of order 2 corresponding to $u=\sqrt{-1}$ and $\sqrt{-t(t-2)}$. For $u=1+\mathrm{e}^{2 \pi i / 3},\{1, u\}$ is a relative integral basis of $L \mid k, h(L)=2$, the H-index is 1 and so $s(u)=1$. A similar analysis applies to $u=\sqrt{-1}$ where $h(L)=6$ and to $u=\sqrt{-t(t-2)}$ where $h(L)=4$. Thus from the co-area formula at $(10)$, we obtain that $\Gamma_{\mathcal{O}}^{+}$has signature $\left(0 ; 2^{(5)}, 3 ; 0\right)$.

Example 2: $[k: \mathbb{Q}]=6, d_{k}=300125, \operatorname{Ram}_{f}(B)=\mathcal{P}_{2}$.

Let $k=\mathbb{Q}(t)$ where $t$ satisfies $t^{6}+t^{5}-7 t^{4}-2 t^{3}+7 t^{2}+2 t-1=0$. Then $k$ is a totally real field of discriminant $300125=5^{3} 7^{4}$ and $h_{k}=1$. Indeed $d_{k}$ is minimal for totally real fields of degree $6 . k$ can also be described as the compositum of the fields $\mathbb{Q}(\sqrt{5})$ and $\mathbb{Q}(\cos 2 \pi / 7)$. There is a unique $k$-prime $\mathcal{P}_{2}$ dividing 2 (of norm $\left.2^{6}\right)$.

Computing a system of fundamental units we obtain that $\left[H: k^{* 2}\right]=\left[R_{f,+}^{*}\right.$ : $\left.R_{f}^{* 2}\right]=2$ generated by the image of 2 . Thus from (10) obtaining an approximation for $\zeta_{k}(2)$ shows that the co-area of $\Gamma_{\mathcal{O}}^{+}$is $2 \pi(111 / 20)$.

Now $2 \cos 2 \pi / m \in k$ for $m=3,4,5,6,7,10,14$. For $m=6,10,14,2+$ $2 \cos 2 \pi / m \notin H$, for $m=3,7, \mathcal{P}_{2}$ splits in the extension $k\left(\mathrm{e}^{2 \pi i / m}\right) \mid k$, for $m=5, \mathcal{P}_{2}$ is inert and for $m=4$, it is ramified. Thus $\mathcal{I}=\{4,5\}$. For elements of order 2 , we note that $\mathcal{P}_{2}$ is ramified in both the extensions $k(\sqrt{-1}) \mid k$ and $k(\sqrt{-2}) \mid k$. Thus the periods will be obtained from $s(u)$ for $u=1+\mathrm{e}^{2 \pi i / 4}, 1+$ $\mathrm{e}^{2 \pi i / 5}, \sqrt{-1}, \sqrt{-2}$.

For $u=1+i$ or $u=i,\{1, u\}$ is a relative integral basis of $k(u)=L$ over $k$. Now $h(L)=3$ and the H-index is readily calculated to be 1 so that, in both cases, from (9) we have $s(u)=3$. For $u=1+\mathrm{e}^{2 \pi i / 5},\{1, u\}$ is again a relative integral basis and (9) applies. In this case $h(L)=2$. There cannot be 
an $\alpha=a+b \mathrm{e}^{2 \pi i / 5} \in R_{L}$ such that $N_{L \mid k}(\alpha)=2$ and $\operatorname{Tr}_{L \mid k}^{2}(\alpha) \in 2 R_{k}$ so that the H-index is 2. Thus $s(u)=1$. For $u=\sqrt{-2},\{1, u\}$ is a relative integral basis and in this case $h(L)=9$ and the H-index is obviously 1 . We thus obtain that $\Gamma_{\mathcal{O}}^{+}$ has signature $\left(0 ; 2^{(9)}, 4^{(3)}, 5 ; 0\right)$.

Example 3: $[k: \mathbb{Q}]=7, d_{k}=41133941, \operatorname{Ram}_{f}(B)=\emptyset$.

Here $k=\mathbb{Q}(t)$ where $t$ satisfies $t^{7}-t^{6}-7 t^{5}+5 t^{4}+13 t^{3}-6 t^{2}-7 t+1=0$. Using Pari we obtain that $R_{k,+}^{*}=R_{k}^{* 2}$ and $h_{k}=1$ so that $\Gamma_{\mathcal{O}}^{+}=\Gamma_{\mathcal{O}}^{1}$. Estimating $\zeta_{k}(2)$ using the small primes (see e.g. $[27, \S 11.2 .4]$ ), we obtain the co-area of $\Gamma_{\mathcal{O}}^{+}=2 \pi V$ where $7.616<V<8.559$. We can only have elements of orders 3 and 2 corresponding to $u=1+\mathrm{e}^{2 \pi i / 3}$ and $u=i$. In both cases, $\{1, u\}$ is a relative integral basis of $L \mid k$. For $u=1+\mathrm{e}^{2 \pi i / 3}, h(L)=7$ and for $u=i, h(L)=10$. From (10) and the area estimate above, it follows that $\Gamma_{\mathcal{O}}^{+}$has signature $\left(0 ; 2^{(10}, 3^{(7)} ; 0\right)$.

Based on the calculations we have made, a reasonable conjecture would appear to be:

Conjecture 5.6. If $\Gamma$ is an arithmetic Fuchsian group of genus 0 , then the degree over $\mathbb{Q}$ of the field of definition of the associated quaternion algebra is at most 7 .

If $\Gamma$ is derived from a quaternion algebra and has genus 0 , then the argument given in the proof of Theorem 4.8 shows that $[k: \mathbb{Q}] \leq 10$. Note that there is a derived group of genus 0 with $[k: \mathbb{Q}]=7$ since, in Example 3 above $\Gamma_{\mathcal{O}}^{+}=\Gamma_{\mathcal{O}}^{1}$. For computations on $\Gamma_{\mathcal{O}}^{1}$, see [39].

\section{The 3-Dimensional PICTURE}

One of our motivations was to understand the analogous situation in dimension 3. As discussed in the Introduction, unlike the 2-dimensional case discussed here, there are arithmetic rational homology 3 -spheres with arbitrarily large injectivity radius. However, the construction of [7] provides commensurable rational homology 3 -spheres. In particular the following are still open.

Question 6.1. Are there infinitely many commensurability classes of arithmetic Kleinian groups $\Gamma$ for which $\mathbf{H}^{3} / \Gamma$ is a rational homology sphere $\left(\right.$ ie $H_{1}(\Gamma ; \mathbb{Q})=$ $0)$ ?

Question 6.2. Are there infinitely many commensurability classes of torsion-free arithmetic Kleinian groups $\Gamma$ for which $\mathbf{H}^{3} / \Gamma$ is a rational homology sphere?

In the spirit of [7], we discuss a conjectural answer to this below. 
6.1. We begin with some notation:

Let $\Gamma$ be a non-cocompact Kleinian (resp. Fuchsian) group acting on $\mathbf{H}^{3}$ (resp. $\mathbf{H}^{2}$ ) with finite co-volume. Let $\mathcal{U}(\Gamma)$ denote the subgroup of $\Gamma$ generated by parabolic elements of $\Gamma$. Note that $\mathcal{U}(\Gamma)$ is visibly a normal subgroup of $\Gamma$, and we may define:

$$
V(\Gamma)=(\Gamma / \mathcal{U}(\Gamma))^{a b} \otimes_{\mathbb{Z}} \mathbb{Q} .
$$

Setting $r(\Gamma)=\operatorname{dim}_{\mathbb{Q}}(V(\Gamma))$, then it follows from standard arguments that $r(\Gamma)$ denotes the dimension of the space of non-peripheral homology.

Let $d$ be a square-free positive integer, and $O_{d}$ be the ring of integers in $\mathbb{Q}(\sqrt{-d})$. The analogue of the modular group here is a family of groups, The Bianchi groups, PSL $\left(2, O_{d}\right)$. In this setting, The Cuspidal Cohomology Problem posed in the 1980's asked which Bianchi groups have $r\left(P S L\left(2, O_{d}\right)\right)=0$. In [46] it was shown that:

Theorem 6.3. $r\left(\operatorname{PSL}\left(2, O_{d}\right)\right)=0$ if and only if

$$
d \in \mathcal{D}=\{1,2,3,5,6,7,11,15,19,23,31,39,47,71\} .
$$

For an ideal $\mathcal{A} \subset O_{d}$ we define the congruence subgroup:

$$
\Gamma_{0}(\mathcal{A})=\mathrm{P}\left\{\left(\begin{array}{ll}
a & b \\
c & d
\end{array}\right): c=0 \bmod \mathcal{A}\right\}
$$

Set $r(\mathcal{A})=r\left(\Gamma_{0}(\mathcal{A})\right)$. Work of Grunewald and Mennicke [16], and Cremona [12], suggest the following: (as discussed in $\S 1$, this is established for $d=2$ in $[6,7])$.

Conjecture 6.4. For each $d \in \mathcal{D}$, there exist infinitely many ideals $\mathcal{A} \subset O_{d}$ such that $r(\mathcal{A})=0$.

For the case of $\mathbb{Z}[i]=O_{1}$, more precise statements can be made. For example, the following seems reasonable.

\section{Conjecture 6.5.}

(a) There exist infinitely many pairs of prime ideals $\left\{\mathcal{P}_{1}, \mathcal{P}_{2}\right\} \subset \mathbb{Z}[i]$ such that $r\left(\mathcal{P}_{1} \mathcal{P}_{2}\right)=0$.

(b) In fact, let $\mathcal{P}=<1+i>$, then there are infinitely many prime ideals $\mathcal{P}_{j} \subset \mathbb{Z}[i]$ with $N \mathcal{P}_{j}=1 \bmod 12$ and $r\left(\mathcal{P} \mathcal{P}_{j}\right)=0$.

As evidence of this, it is shown in [12] that of all integral ideals $\mathcal{A} \subset \mathbb{Z}[i]$ with norm $\leq 500$ only 76 give rise to groups with $r(\mathcal{Q})>0$. With reference to Conjecture 6.5(b) [12] shows that this holds for prime ideals of norms 13, 37, 61, and 73 , but fails for a prime of norm 97 . 
As in [7], the relevance of these conjectures to the cocompact setting follows from the Jacquet-Langlands correspondence (see [18] and also [7] Theorem 3.3). A consequence of this is.

Theorem 6.6. Let $B$ be a division algebra of quaternions over $\mathbb{Q}(\sqrt{-d})$ with the set of primes ramifying $B$ being, $\left\{\mathcal{P}_{1}, \ldots, \mathcal{P}_{2 r}\right\}$. Let $\mathcal{O}$ be a maximal order of $B$, and $\Gamma=\Gamma_{\mathcal{O}}^{1}$. If $\Gamma^{a b}$ is infinite, then $r\left(\mathcal{P}_{1} \ldots \mathcal{P}_{2 r}\right) \neq 0$.

A corollary of this result and Conjecture 6.5(b) is the following. We remark that the prime $\mathcal{P}$ in Conjecture $6.5(\mathrm{~b})$ is chosen so that the resultant groups $\Gamma_{\mathcal{O}}^{1}$ are torsion-free.

Corollary 6.7. If Conjecture 6.5(b) holds, then Question 6.2 has a positive answer.

Remark: Theorem 4.8 shows that there are just two arithmetic integral homology 2 -spheres. There are examples of arithmetic integral homology 3 -spheres, but only finitely many examples are known to the authors.

As an example of an arithmetic integral homology 3-sphere one can take the 3 -fold cyclic branched cover of the $(-2,3,7)$-pretzel knot. The invariant trace field is $\mathbb{Q}(\theta)$ where $\theta$ has minimal polynomial $x^{6}-x^{5}-x^{4}+2 x^{3}-2 x^{2}-x+1$. This generates a field of signature $(4,1)$ and discriminant -104483 . The algebra $B$ is ramified at all the real places and unramified at all other places. All of this can be checked using Snap (see [11]).

\section{REFERENCES}

[1] M. Alsina and P. Bayer, Quaternion orders, quadratics forms and Shimura curves, C.R.M. Monograph Series 22, Providence R.I. (2004).

[2] I. Agol, Finiteness of arithmetic Kleinian reflection groups, preprint.

[3] P. Ackermann, M. Näätänen and G. Rosenberger, The arithmetic Fuchsian groups with signature (0;2, 2, 2, q), Research and Expositions in Math. 27 (2003), 1-9.

[4] C. Baer, Klassifikation arithmetischer Fuchsscher Gruppen der Signatur $\left(0 ; e_{1}, e_{2}, e_{3}, e_{4}\right)$, Dissertation, Univ. Dortmund 2001.

[5] A. Borel, Commensurability classes and volumes hyperbolic three-manifolds, Ann. Scuola Norm. Sup. Pisa 8 (1981), 1-33.

[6] N. Boston and J. S. Ellenberg, Pro-p groups and towers of rational homology spheres, Geometry and Topology 10 (2006), 331-334.

[7] F. Calegari and N. Dunfield, Automorphic forms and rational homology 3-spheres, Geometry and Topology 10 (2006), 295-329.

[8] T. Chinburg and E. Friedman, The finite subgroups of maximal arithmetic subgroups of $\operatorname{PSL}(2, \mathbb{C})$, Ann. Inst. Fourier 50, (1999) 1765-1798.

[9] H. Cohen et al, Pari, Freeware available at http://pari.math.u-bordeaux.fr.

[10] J. H. Conway and S. Norton, Monstrous moonshine, Bull. L. M. S. 11 (1979), 308-339.

[11] D. Coulsen, O. A. Goodman, C. D. Hodgson and W. D. Neumann, Computing arithmetic invariants of 3-manifolds, Experimental J. Math. 9 (2000), 127-152. 
[12] J. E. Cremona, Hyperbolic tessellations, modular symbols and elliptic curves over complex quadratic fields, Composito Math. 51 (1984) 275-323.

[13] M. Eichler, Zur Zahlentheorie der Quaternionenalgebren, J. Reine Angew. Math. 195, (1955) 127-155.

[14] M. Eichler, Lectures on Modular Correspondences, Tata Institute Notes (1955-56).

[15] S. S. Gelbart and H. Jacquet, A relation between automorphic representations of GL(2) and GL(3), Ann. Sci. Ecole Norm. Sup. 11 (1978), 471-542.

[16] F. Grunewald and J. Mennicke, $\mathrm{SL}_{2}(\mathrm{O})$ and elliptic curves, Manuscript Bielefeld.

[17] H. Helling, On the commensurability class of the rational modular group, J. London Math. Soc. 2, (1970) 67-72.

[18] H. Jacquet and R. P. Langlands, Automorphic Forms on GL(2), L.N.M. 114, Springer (1970).

[19] S. Johansson, Genera of arithmetic Fuchsian groups, Acta Arithmetica 86 (1998), pp. 171-191.

[20] P. G. Kluit, The normalizer of $\Gamma_{0}(N)$, Modular Functions of one variable V, L.N.M. 601, 239-248 Springer Berlin (1977).

[21] S. Lang, Algebraic Number Theory, Grad. Texts in Math. 110 Springer (1986).

[22] D. D. Long and A. W. Reid, Simple quotients of hyperbolic 3-manifold groups, Proc. A. M. S. 126 (1998), 877-880.

[23] S. Louboutin, Majorations explicites de $|L(1, \chi)| I I$, Comptes Rendus Acad. Sci. Paris Sér I Math. 323 (1996) 443-446.

[24] C. Maclachlan, Groups of units of zero ternary quadratic forms, Proc. Royal Soc. Edinburgh 88 (1981) 141-157.

[25] C. Maclachlan, Torsion in arithmetic Fuchsian groups, J. London Math. Soc (2) 73 (2006) $14-30$.

[26] C. Maclachlan, Torsion in maximal arithmetic Fuchsian groups, to appear in Conf. on Combinatorial Group Theory (Rosenberger Festschrift) Ed. B. Fine et al, AMS Contemporary Math. Series.

[27] C. Maclachlan and A. W. Reid, The Arithmetic of Hyperbolic 3-Manifolds, Grad. Texts in Math. 219 Springer (2003).

[28] C. Maclachlan and G. Rosenberger, Two-generator arithmetic Fuchsian groups II, Math. Proc. Camb. Phil. Soc. 111 (1992), 7-24.

[29] C. Maclachlan and G. Rosenberger, Commensurability classes of 2-generator Fuchsian groups, Lecture Note Series Vol 173, London Maths. Soc (1992) 171-189.

[30] J. Mennicke, On the groups of units of ternary quadratic forms with rational coefficients, Proc. Royal Soc, Edinburgh Sect. A 67 (1963-7) 309-352.

[31] W. Narkiewicz Elementary and Analytic Theory of Algebraic Numbers, Second edition, Polish Scientific Publisher, Warsaw 1990.

[32] W. Neumann and A.W. Reid Arithmetic of hyperbolic manifolds, in Topology '90, De Gruyter, Berlin (1992) 273-310.

[33] V. V. Nikulin, On the classification of arithmetic groups generated by reflections in Lobachevskii spaces, Math. USSR-Izv. 18 (1982), 99-123.

[34] A. Odlyzko, Some analytic estimates of class numbers and discriminants, Invent. Math. 29 (1975), 275-286.

[35] A.P. Ogg, Automorphismes des Courbes Modulaires, Seminaire Delange-Pisot, Poitou, 7, (1974).

[36] A.P. Ogg, Modular functions, Proc. Symp. Pure Maths. 37 (1980) 521-532.

[37] A.P. Ogg, Über die Automorphismengruupe von $X_{0}(N)$, Math. Ann. 228 (1977) 279-292.

[38] A. P. Ogg, Real points on Shimura curves, Arithmetic and Geometry I, Progr. Math. 35, Birkhauser, (1983) 277-303. 
[39] V. Schneider, Die elliptischen Fixpunkte zu Modulgruppen in Quaternionenschiefkörper, Math. Annalen 217 (1975), pp. 29-45.

[40] G. Shimura, Construction of class fields and zeta functions of algebraic curves, Ann. Math. 85 (1967) 58-159.

[41] K. Takeuchi, Commensurability Classes of arithmetic triangle groups, J. Fac. Sci. Univ. Tokyo 24 (1977), 201-222.

[42] K. Takeuchi, Arithmetic Fuchsian groups of signature $(1 ; e)$, J. Math. Soc. Japan 35 (1983), 381-407.

[43] J. G. Thompson, A finiteness theorem for subgroups of $\operatorname{PSL}(2, \mathbb{R})$ which are commensurable to $\operatorname{PSL}(2, \mathbb{Z})$, Proc. Symp. Pure Math A.M.S. 37 (1980), 533-555.

[44] M-F Vigneras, Arithmétique des Algèbres de Quaternions, L.N.M. 800, Springer (1980).

[45] M-F Vigneras, Quelques remarques sur la conjecture $\lambda_{1} \geq \frac{1}{4}$, Seminar on number theory, Paris 1981-82, Progr. Math., 38 Birkhauser, (1983) 321-343.

[46] K. Vogtmann, Rational homology of Bianchi groups, Math. Annalen 272 (1985), 399-419.

[47] P. Zograf, A spectral proof of Rademacher's conjecture for congruence subgroups of the modular group, J. Reine Angew. Math. 414 (1991), 113-116.

[48] Lists of number fields, available at ftp://megrez.math.u-bordeaux.fr/pub/numberfields/.

\section{D. Long}

Department of Mathematics

University of California

Santa Barbara, CA 93106, U.S.A

E-mail: Darren.Long@math.ucsb.edu

C. Maclachlan

Department of Mathematical Sciences

University of Aberdeen

Aberdeen, AB24 3UE, U.K.

E-mail: C.Maclachlan@maths.abdn.ac.uk

A. W. Reid

Department of Mathematics

University of Texas

Austin, TX 78712, U.S.A.

E-mail: areid@math.utexas.edu 